\title{
Enumeration of small Wilf classes avoiding 1324 and two other 4-letter patterns
}

\author{
David Callan \\ Department of Statistics \\ University of Wisconsin \\ Madison, WI 53706, USA \\ email: callan@stat.wisc.edu \\ and \\ TOUfiK MANsour \\ Department of Mathematics \\ University of Haifa \\ 3498838 Haifa, Israel \\ email: tmansour@univ.haifa.ac.il
}

(Received: May 3, 2017, and in revised form June 26, 2017.)

\begin{abstract}
Recently, it has been determined that there are 242 Wilf classes of triples of 4-letter permutation patterns by showing that there are 32 non-singleton Wilf classes. Moreover, the generating function for each triple lying in a non-singleton Wilf class has been explicitly determined. In this paper, toward the goal of enumerating avoiders for the singleton Wilf classes, we obtain the generating function for all but one of the triples containing 1324. (The exceptional triple is conjectured to be intractable.) Our methods are both combinatorial and analytic, including generating trees, recurrence relations, and decompositions by left-right maxima. Sometimes this leads to an algebraic equation for the generating function, sometimes to a functional equation or a multi-index recurrence amenable to the kernel method.
\end{abstract}

Mathematics Subject Classification(2010). 05A05, 05A15.

Keywords: pattern avoidance, generating function, generating tree, kernel method.

\section{Introduction}

In recent decades, pattern avoidance has received a lot of attention. It has a prehistory in the work of MacMahon [21] and Knuth [17], but the current interest was sparked by a paper of Simion and Schmidt 25. They thoroughly analyzed 3-letter patterns in permutations, including a bijection between 123- and 132-avoiding permutations, thereby explaining the first (nontrivial) instance of what is, in modern terminology, a Wilf class. Since then the problem has been addressed on several other discrete structures, such as compositions, $k$-ary words, and set partitions; see, e.g., the texts [15,22] and references contained therein.

Permutations avoiding a single 4-letter pattern have been well studied (see, e.g., [1,22, 28, 30]), and the latter form 7 symmetry classes and 3 Wilf classes. As for pairs of 4-letter patterns, there are 56 symmetry classes, for all but 5 of which the avoiders have been enumerated [1]. Le [20] established that these 56 symmetry classes form 38 distinct Wilf classes. 
The $\left(\begin{array}{c}24 \\ 3\end{array}\right)=2024$ triples of 4-letter patterns split into 317 symmetry classes. It is known [12, 13, that the 317 symmetry classes split into 242 Wilf classes, 32 of which are large (a Wilf class is called large if it contains at least two symmetry classes, otherwise, it is small) and the large Wilf classes are all explicitly enumerated in [12,13] (each has an algebraic generating function).

Our goal here is to enumerate (with one exception, see [26, Seq. A257562]) all the small Wilf classes that contain the pattern 1324. Running the INSENC algorithm (regular insertion encoding, see [3,29]) over all the 210 small Wilf classes determines the generating function for 126 of them [10, Appendix]. The remaining small classes that contain 1324 are listed in Table 1 along with their generating functions, where the numbering follows that of Table 2 in the appendix to [5], based on lex order of counting sequences.

In this abbreviated paper, we prove a selection of the results in Table 1, illustrating a variety of methods. The full paper, containing all proofs, is posted to the ArXiv [10. Section 2 contains some preliminary remarks, and Section 3 gives our selection of proofs.

Table 1: Small Wilf classes of three 4-letter patterns not counted by INSENC that include the pattern 1324

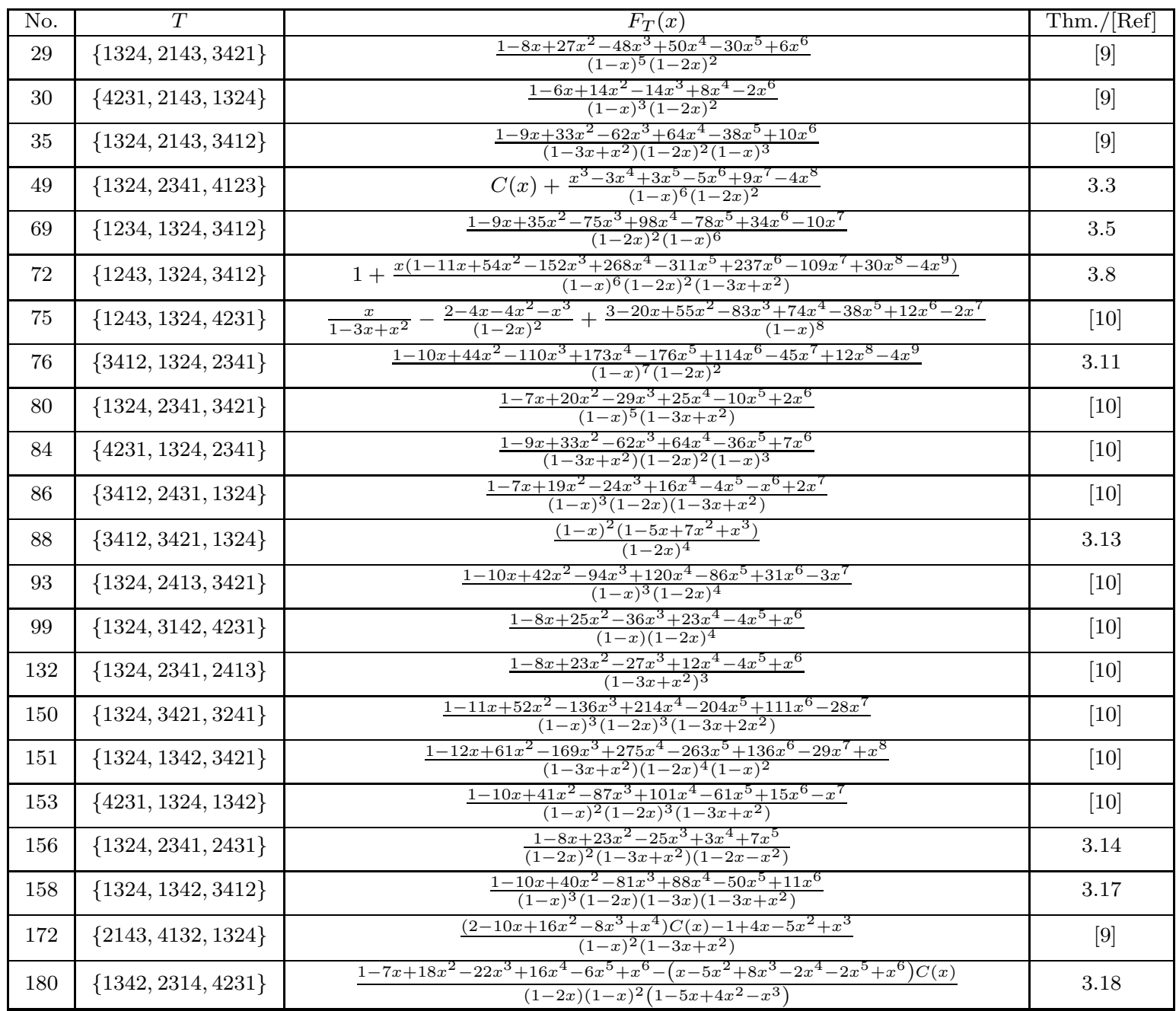




\begin{tabular}{|c|c|c|c|}
\hline No. & $T$ & $F_{T}(x)$ & Thm./[Ref] \\
\hline 184 & $\{1324,2431,3241\}$ & $\frac{1-8 x+24 x^{2}-32 x^{3}+19 x^{4}-3 x^{5}}{(1-x)(1-2 x)\left(1-3 x+x^{2}\right)^{2}}$ & {$[10]$} \\
\hline 187 & $\{1324,2314,2431\}$ & $\frac{1-9 x+31 x^{2}-49 x^{3}+34 x^{4}-7 x^{5}}{\left(1-3 x+x^{2}\right)^{2}(1-2 x)^{2}}$ & {$[10$} \\
\hline 193 & $\{1324,2431,3142\}$ & $\frac{x-1+\left(x^{2}-5 x+2\right) C(x)}{1-3 x+x^{2}}$ & {$[3.19$} \\
\hline 195 & $\{1324,2341,1243\}$ & $\frac{\left(1-7 x+19 x^{2}-25 x^{3}+13 x^{4}+4 x^{5}-5 x^{6}+x^{7}\right) C(x)-1+7 x-19 x^{2}+23 x^{3}-7 x^{4}-7 x^{5}+4 x^{6}}{x(1-x)^{2}\left(1-3 x+x^{2}\right)\left(1-x-x^{2}\right)}$ & {$[10$} \\
\hline 210 & $\{1243,1324,2431\}$ & $\frac{\left(1-6 x+13 x^{2}-11 x^{3}+4 x^{4}\right)}{x^{2}(1-x)^{2}}$ & {$[3.20$} \\
\hline 211 & $\{1234,1324,2341\}$ & $\frac{\left(1-4 x+5 x^{2}-3 x^{3}\right) C(x)-\left(1-4 x+6 x^{2}-4 x^{3}\right)}{x(1-x)^{2}(1-2 x)}$ & {$[3.21$} \\
\hline 212 & $\{1324,2413,2431\}$ & $1+\frac{x\left(1-4 x+4 x^{2}-x^{3}-x\left(1-4 x+2 x^{2}\right) C(x)\right)}{\left(1-3 x+x^{2}\right)\left(1-3 x+x^{2}-x(1-2 x) C(x)\right)}$ & {$[3.22$} \\
\hline 213 & $\{2431,1324,1342\}$ & $\frac{\left(1-5 x+8 x^{2}-5 x^{3}\right) C(x)-1+4 x-4 x^{2}+x^{3}}{x^{2}(1-2 x)}$ & {$[10$} \\
\hline 227 & $\{2143,1432,1324\}$ & $\frac{1-6 x+12 x^{2}-12 x^{3}+6 x^{4}-x^{5}-x^{2}\left(1-x+x^{2}\right)^{2} C(x)}{1-7 x+16 x^{2}-19 x^{3}+11 x^{4}-2 x^{5}-x^{6}}$ & {$[9]$} \\
\hline 231 & $\{1324,1342,2341\}$ & $\frac{(1-3 x)(1-2 x-x C(x))}{(1-4 x)\left(1-3 x+x^{2}\right)}$ & {$[3.25$} \\
\hline 237 & $\{1432,1324,1243\}$ & $?$ & {$[26]$} \\
\hline 241 & $\{1432,1324,1243\}$ & $\frac{\left(v_{-}-1\right)\left(v_{+}-1\right)\left(\left(v_{-}+v_{+}\right)\left(v_{-}^{2}+v_{+}^{2}-x^{2}\right)+(x-1)\left(v_{-}^{2}+v_{+}^{2}+v_{-} v_{+}\right)\right)}{x-\left(v_{-}-1\right)\left(v_{+}-1\right)\left(v_{-}^{2}+v_{+}^{2}+v_{-} v_{+}+x\left(v_{-}+v_{+}+2-x\right)\right)}$ & {$[3.27$} \\
\hline
\end{tabular}

\section{Preliminaries}

We say a permutation is standard if its support set is an initial segment of the positive integers, and for a permutation $\pi$ whose support is any set of positive integers, $\operatorname{St}(\pi)$ denotes the standard permutation obtained by replacing the smallest entry of $\pi$ by 1 , the next smallest by 2 , and so on. Typically, for a given triple $T$, we consider cases and analyze the structure of a $T$-avoider in each case to the point where we say that $T$-avoiders have such and such a form in that case. It is always to be understood that we are also asserting, without explicit mention, that a permutation of the specified form is a $T$-avoider, and this enables us to determine the various "contributions" to the generating function $F_{T}(x)$ for $T$-avoiders, yielding an equation for $F_{T}(x)$. The equation may be an explicit expression for $F_{T}(x)$ or an algebraic or functional equation. For all but one symmetry class, the generating function turns out to be algebraic of degree $\leq 4$. For the exceptional class (Case 237, where $\{1432,1324,1243\}$ and $\{4123,4231,4312\}$ are representative triples), the generating function is conjectured not to satisfy any ADE (algebraic differential equation), see [2] and [26, Seq. A257562].

A permutation $\pi$ expressed as $\pi=i_{1} \pi^{(1)} i_{2} \pi^{(2)} \cdots i_{m} \pi^{(m)}$ where $i_{1}<i_{2}<\cdots<i_{m}$ and $i_{j}>$ $\max \left(\pi^{(j)}\right)$ for $1 \leq j \leq m$ is said to have $m$ left-right maxima (at $\left.i_{1}, i_{2}, \ldots, i_{m}\right)$. Given nonempty sets of numbers $S$ and $T$, we will write $S<T$ to mean $\max (S)<\min (T)$ (with the inequality vacuously holding if $S$ or $T$ is empty). In this context, we will often denote singleton sets simply by the element in question. Also, for a number $k, S-k$ means the set $\{s-k: s \in S\}$.

Throughout, $C(x)=\frac{1-\sqrt{1-4 x}}{2 x}$ denotes the generating function for the Catalan numbers $C_{n}:=$ $\frac{1}{n+1}\left(\begin{array}{c}2 n \\ n\end{array}\right)=\left(\begin{array}{c}2 n \\ n\end{array}\right)-\left(\begin{array}{c}2 n \\ n-1\end{array}\right)$. As is well known [1, 17], $C(x)$ is the generating function for $\left(\left|S_{n}(\pi)\right|\right)_{n \geq 0}$ where $\pi$ is any one of the six 3-letter patterns. The identity $C(x)=\frac{1}{1-x C(x)}$ or, equivalently, $x C(x)^{2}=$ $C(x)-1$ is used to simplify some of our results. Also throughout, $L(x)=\frac{1-x}{1-2 x}$ denotes the generating function for $\{213,231\}$-avoiders (resp. $\{213,123\}$-avoiders, resp. $\{132,123\}$-avoiders), see [25], and $K(x), K^{\prime}(x)$, etc., are variously used for other known generating functions. 


\section{Proofs}

3.1 Case 49: $\{1324,2341,4123\}$

In order to study this case, we need the following lemmas.

Lemma 3.1 Let $T=\{1324,2341,4123\}$. The generating function for the number of permutations $(n-1) \pi^{\prime} n \pi^{\prime \prime} \in S_{n}(T)$ is given by

$$
H(x)=\frac{x^{3} C(x)^{3}}{1-x}+\frac{x^{2}}{1-x}+\frac{x^{4}}{(1-x)(1-2 x)}+\frac{x^{5}}{(1-x)^{4}} .
$$

Proof. Let us write an equation for $H(x)$. Let $\pi=(n-1) \pi^{\prime} n \pi^{\prime \prime} \in S_{n}(T)$. If $n=2$, then we have a contribution of $x^{2}$. So assume $n>2$, and consider the two cases, either $n-2$ belongs to $\pi^{\prime}$ or to $\pi^{\prime \prime}$.

- $n-2$ belongs to $\pi^{\prime}$ : If $\pi^{\prime \prime}=\emptyset$ then we have a contribution of $x^{3}(L(x)-1)=\frac{x^{3}}{1-2 x}$, see [25]. So, we can assume that $\pi^{\prime \prime} \neq \emptyset$. If $\pi^{\prime}$ has a letter between $n-1$ and $n-2$, then $\pi$ can be written as

$$
\pi=(n-1)(i-1)(i-2) \cdots j(n-2)(j-1)(j-2) \cdots 1 n(n-3)(n-4) \cdots i,
$$

which is counted by $\frac{x^{5}}{(1-x)^{3}}$. Otherwise, $\pi^{\prime}$ has no letter between $n-1$ and $n-2$, which gives a contribution of $x H(x)-\frac{x^{3}(1-x)}{1-2 x}$.

- $n-2$ belongs to $\pi^{\prime \prime}$ : In this case, we have a contribution of $x(C(x)-1-x C(x))=x^{3} C(x)^{3}$, where $C(x)$ counts the $\{123\}$-avoiders.

Hence, by adding all the contributions, we have

$$
H(x)=x^{2}+\frac{x^{3}}{1-2 x}+\frac{x^{5}}{(1-x)^{3}}+x H(x)-\frac{x^{3}(1-x)}{1-2 x}+x^{3} C(x)^{3},
$$

which completes the proof.

Lemma 3.2 Let $T=\{1324,2341,4123\}$. The generating function for the number of $T$-avoiders with exactly 2 left-right maxima is given by

$$
G_{2}(x)=\frac{1}{1-x}\left(x^{4} C(x)^{5}+\frac{x^{5}}{(1-x)^{5}}+\frac{x^{5}}{(1-x)^{4}}+\frac{x^{4}}{1-2 x}+H(x)\right),
$$

where $H(x)$ is given in Lemma 3.1.

Proof. Let us write an equation for $G_{2}(x)$. Let $\pi=i \pi^{\prime} n \pi^{\prime \prime} \in S_{n}(T)$ be a permutation with exactly two left-right maxima. We consider the following cases:

- $i=n-1$ : We have a contribution of $H(x)$ as defined in Lemma 3.1. So from now, we assume that $\pi^{\prime \prime}$ contains the letter $n-1$.

- $\pi^{\prime \prime}=(n-1) \pi^{\prime \prime \prime}$ : We have a contribution of $x G_{2}(x)$. So we can assume that there is at least one letter between $n$ and $n-1$. Since $\pi^{\prime \prime}$ avoids 1324 and 4123, we see that there is at most one letter between $n$ and $n-1$ greater than $i$. 
- if there is a letter in $n \pi^{\prime \prime}$ between $n$ and $n-1$ that it is greater than $i$, then $\pi$ can be written as $\pi=i \pi^{\prime} n k \beta(n-1) \cdots(k+1) \alpha$ such that $\beta<i$ and $\alpha>i$. By considering whether $\beta$ is empty or not, we obtain the contributions $\frac{x^{4}}{1-x} L(x)^{2}$ and $\frac{x^{5}}{(1-x)^{4}}$, respectively.

- Thus, we can assume that $\pi=\pi^{\prime} n \beta(n-1) \alpha$ such that $\alpha$ contains the subsequence $(n-$ 1) $(n-2) \cdots(i+1), \beta<i$ and $\beta$ is decreasing (since $\pi$ avoids 4123 , and $\beta$ is not empty). If $\beta=e e^{\prime} \beta^{\prime}$, then $e>e^{\prime}>\beta^{\prime}$ and $\pi^{\prime}>e^{\prime}$. If $\pi^{\prime}$ has a letter between $e$ and $e^{\prime}$, then it is easy to see that the contribution is given by $\frac{x^{4+d}}{(1-x)^{4}}$, where $d$ is the number of letters in $\pi^{\prime \prime}$ that are greater than $i$. Otherwise, the contribution is given by $x^{3+d} C(x)^{3+d}$, where $d$ is the number of letters in $\pi^{\prime \prime}$ that are greater than $i$. Therefore, we have a contribution of

$$
\sum_{d \geq 1} \frac{x^{4+d}}{(1-x)^{4}}+\sum_{d \geq 1} x^{3+d} C(x)^{3+d}=\frac{x^{5}}{(1-x)^{5}}+\frac{x^{4} C(x)^{4}}{1-x C(x)} .
$$

Hence, the various contributions yield

$$
G_{2}(x)=x G_{2}(x)+x^{4} C(x)^{5}+\frac{x^{5}}{(1-x)^{5}}+\frac{x^{5}}{(1-x)^{4}}+\frac{x^{4}}{1-2 x}+H(x),
$$

which completes the proof.

Theorem 3.3 Let $T=\{1324,2341,4123\}$. Then

$$
F_{T}(x)=C(x)+\frac{x^{3}-3 x^{4}+3 x^{5}-5 x^{6}+9 x^{7}-4 x^{8}}{(1-x)^{6}(1-2 x)^{2}} .
$$

Proof. Let $G_{m}(x)$ be the generating function for $T$-avoiders with $m$ left-right maxima. Clearly, $G_{0}(x)=1$ and $G_{1}(x)=x F_{\{123\}}(x)=x C(x)$, see [17]. By Lemmas 3.1 and 3.2, we have

$$
G_{2}(x)=\frac{1}{1-x}\left(x^{4} C(x)^{5}+\frac{x^{5}}{(1-x)^{5}}+\frac{x^{5}}{(1-x)^{4}}+\frac{x^{4}}{1-2 x}+H(x)\right),
$$

where

$$
H(x)=\frac{x^{3} C(x)^{3}}{1-x}+\frac{x^{2}}{1-x}+\frac{x^{4}}{(1-x)(1-2 x)}+\frac{x^{5}}{(1-x)^{4}} .
$$

Now, let us write an equation for $G_{3}(x)$. Let $\pi=i_{1} \pi^{\prime} i_{2} \pi^{\prime \prime} n \pi^{\prime \prime \prime} \in S_{n}(T)$ with exactly 3 left-right maxima $\left(i_{1}, i_{2}, n\right)$. Since $\pi$ avoids 1324 and 2341, then $\pi^{\prime}>\pi^{\prime \prime}$ and $\pi^{\prime \prime \prime}=\beta \alpha$ such that $\beta>i_{2}>\alpha>i_{1}$. By considering cases based on whether or not $\alpha$ or $\beta$ is empty, we obtain the contributions $x^{3} L(x)^{2}$, $x^{3}(L(x)-1) L(x) /(1-x), x^{3}(L(x)-1) L(x) /(1-x)$, and $x^{5} /(1-x)^{4}$. Hence,

$$
G_{3}(x)=x^{3} L(x)^{2}+\frac{2 x^{3}}{1-x}(L(x)-1) L(x)+\frac{x^{5}}{(1-x)^{4}} .
$$

By a similar method, we obtain

$$
G_{4}(x)=x^{4}(L(x)+(L(x)-1) /(1-x))^{2} .
$$


Now, let us write an equation for $G_{m}(x)$ where $m \geq 5$. Let $\pi=i_{1} \pi^{(1)} i_{2} \pi^{(2)} \cdots i_{m} \pi^{(m)} \in S_{n}(T)$ with exactly $m$ left-right maxima. Since $\pi$ avoids $T$, we see that $\pi^{(s)}=\emptyset$ for all $s=3,4, \ldots, m-1$, $\pi^{(1)}>\pi^{(2)}$ and $\pi^{(m)}=\beta \alpha$ such that $\beta>i_{m-1}>\alpha>i_{m-2}$. Thus, $G_{m}(x)=x G_{m-1}(x)$ for all $m \geq 5$. Therefore,

$$
F_{T}(x)-1-x C(x)-G_{2}(x)-G_{3}(x)=\frac{G_{4}(x)}{1-x} .
$$

By substituting the expressions for $G_{2}(x), G_{3}(x), G_{4}(x)$ and simplifying, we obtain the stated generating function.

\subsection{Case 69: $\{1234,1324,3412\}$}

A permutation $\pi=\pi_{1} \pi_{2} \cdots \pi_{n} \in S_{n}$ determines $n+1$ positions, called sites, between its entries. The sites are denoted $1,2, \ldots, n+1$ left to right. In particular, site $i$ is the space between $\pi_{i-1}$ and $\pi_{i}, 2 \leq i \leq n$. Site $i$ in $\pi$ is said to be active (with respect to $T$ ) if, by inserting $n+1$ into $\pi$ in site $i$, we get a permutation in $S_{n+1}(T)$; otherwise, it is inactive.

Say $j$ is an ascent index for a permutation $\pi=\pi_{1} \pi_{2} \cdots \pi_{n}$ of $[n]$ if $\pi_{j}<\pi_{j+1}$, in which case, $\pi_{j}$ is an ascent bottom.

To construct the generating forest for $T$-avoiders, we first specify the labels. For $n \geq 2$, define the label of $\pi \in S_{n}(T)$ to be $(k, s)$, where $k$ is the number of active sites in $\pi$ and $s$ is the number of active sites greater than the largest ascent index (LAI for short) with LAI taken to be 0 if there are no ascents, that is, if $\pi$ is decreasing.

For instance, the active sites for $\pi=12$ are $\{1,2,3\}$ and $\mathrm{LAI}=1$, so the label for 12 is $(3,2)$. Also, 12 has three children 312,132 and 123 with active sites $\{1,3,4\},\{1,2,3\}$ and $\{1,2,3\}$, respectively, and $\mathrm{LAI}=2,1,2$, respectively; hence labels $(3,2),(3,2)$, and $(3,1)$. Similarly, all 3 sites for 21 are active and LAI $=0$, so its label is $(3,3)$, and it has three children 321, 231 and 213 with active sites $\{1,2,3,4\}$ in all three cases, and LAI $=0,1,2$, respectively; hence labels $(4,4),(4,3)$, and $(4,2)$. An avoider $\pi \in S_{n}(T)$ has a label $(k, s)$ with $k=s$ only if $\pi$ is decreasing, in which case $k=s=n+1$. Otherwise, $0 \leq s<k$.

Proposition 3.4 The roots for the generating forest $\mathcal{T}$ of $S_{n}(T)$ are 12 and 21 with labels $(3,2)$ and $(3,3)$ respectively, and the succession rules for the labels of children, in order of increasing insertion site, are given by

$$
\begin{aligned}
(k, s) & \rightsquigarrow(1,0)(2,0) \ldots(k, 0) & & \text { for } s=0 \text { and } k \geq 1, \\
& \rightsquigarrow(2,1)(2,0)(3,0) \ldots(k-1,0)(k, 1) & & \text { for } s=1 \text { and } k \geq 2, \\
& \rightsquigarrow(s+1, s)(3,1)(4,1) \ldots(k-s+1,1) & & \text { for } 2 \leq s \leq k-1, \\
& \rightsquigarrow(k-s+2,2)(k-s+2,1)(k-s+3,1) \ldots(k, 1) & & \text { for } s=k,
\end{aligned}
$$

As an example (bullets denote active sites, an underscore denotes last ascent bottom), the label of $\pi=\bullet \cdot \cdot \underline{2} \bullet 6 \bullet 541 \in S_{6}(T)$ is $(k, s)=(4,2)$; its children are $\bullet 73 \underline{2} \cdot 6 \cdot 541, \bullet 3 \bullet 7 \underline{2} \cdot 6541$, $\cdot 3 \cdot \underline{2} \cdot 7 \cdot 6541, \cdot 3 \cdot 2 \cdot \underline{6} \bullet 7541$, in that order, with labels $(3,2),(3,1),(4,2),(4,1)$ respectively.

The proof of Proposition 3.4 is based on induction by a routine verification of cases, and is left to the reader. We note a few properties of the active sites for $\pi \in S_{n}(T)$. Site 1 is always active. If site 
$n+1$ is active, then so is site $n$. The active sites always form either an interval of integers (necessarily an initial segment of the positive integers) or a pair of intervals of integers. In the latter case, at least one of the intervals is of length 1 . For example, $532416 \in S_{6}(T)$ has active sites $\{1,4,5,6\}$ and $5342761 \in S_{7}(T)$ has active sites $\{1,2,5\}$.

Enumeration: Let $A_{k, s}=A_{k, s}(t)$ be the generating function for the number of vertices labeled $(k, s)$ in level $n$ in the generating forest $\mathcal{T}$, where the roots 12 and 21 are at level 2. Define

$$
A(u, v)=A(t ; u, v)=\sum_{k \geq 1, s \geq 0} A_{k, s} u^{k} v^{s}, \quad L(u, v)=\frac{u^{3} v^{3} t^{2}}{1-u v t} .
$$

Proposition 3.4 leads to

$$
\begin{aligned}
A(u, v) & =t^{2} u^{3} v^{2}(1+v)+\frac{u t}{1-u}(A(1,0)-A(u, 0))+\left.v t \frac{d}{d v} A(u, v)\right|_{v=0} \\
& +\frac{t}{1-u}\left(\left.u^{2} \frac{d}{d v} A(1, v)\right|_{v=0}-\left.\frac{d}{d v} A(u, v)\right|_{v=0}\right)+u t A(1, u v)-u t L(1, u v)-u t A(1,0) \\
& +\frac{v u^{3} t}{1-u}\left(A(1,1)-A(1,0)-\left.\frac{d}{d v} A(1, v)\right|_{v=0}-L(1,1)\right) \\
& -\frac{u v t}{1-u}\left(A(u, 1)-A(u, 0)-\left.\frac{d}{d v} A(u, v)\right|_{v=0}-L(u, 1)\right) \\
& +u^{2} v^{2} t\left(A(u, 1 / u)-A(u, 0)-\left.\frac{1}{u} \frac{d}{d v} A(u, v)\right|_{v=0}-L(1,1)\right) \\
& +\frac{u^{4} v^{2} t^{3}}{(1-v)(1-u t)}-\frac{u^{4} v^{5} t^{3}}{(1-v)(1-u v t)}
\end{aligned}
$$

By substituting $v=1 / u$ into (1), we obtain

$$
A(u, 1 / u)=\frac{t\left(t-u^{2} t+u A(1,1)-A(u, 1)\right)}{(1-u)(1-x)},
$$

which implies

$$
\begin{aligned}
A(u, v) & =t^{2} u^{3} v^{2}(1+v)+\frac{u t}{1-u}(A(1,0)-A(u, 0))+\left.v t \frac{d}{d v} A(u, v)\right|_{v=0} \\
& +\frac{t}{1-u}\left(\left.u^{2} \frac{d}{d v} A(1, v)\right|_{v=0}-\left.\frac{d}{d v} A(u, v)\right|_{v=0}\right)+u t A(1, u v)-u t L(1, u v)-u t A(1,0) \\
& +\frac{v u^{3} t}{1-u}\left(A(1,1)-A(1,0)-\left.\frac{d}{d v} A(1, v)\right|_{v=0}-L(1,1)\right) \\
& -\frac{u v t}{1-u}\left(A(u, 1)-A(u, 0)-\left.\frac{d}{d v} A(u, v)\right|_{v=0}-L(u, 1)\right) \\
& +u^{2} v^{2} t\left(\frac{t\left(t-u^{2} t+u A(1,1)-A(u, 1)\right)}{(1-u)(1-x)}-A(u, 0)-\left.\frac{1}{u} \frac{d}{d v} A(u, v)\right|_{v=0}-L(1,1)\right) \\
& +\frac{u^{4} v^{2} t^{3}}{(1-v)(1-u t)}-\frac{u^{4} v^{5} t^{3}}{(1-v)(1-u v t)}
\end{aligned}
$$


Substitute $v=0$ into (21) and into the derivative of (2) with respect to $v$. Solve the resulting system for the variables $\left.\frac{d}{d v} A(u, v)\right|_{v=0}$ and $\left.\frac{d}{d v} A(1, v)\right|_{v=0}$ to get

$$
\begin{aligned}
\left.\frac{d}{d v} A(u, v)\right|_{v=0} & =\frac{2 u t-2 u+1}{1-2 t} A(u, 0)-\frac{u t}{(1-u)(1-2 t)} A(u, 1)-\frac{(1-u) u t}{1-2 t} A(1,0) \\
& +\frac{u^{3} t}{(1-u)(1-2 t)}-\frac{u^{3} t^{3}}{(1-t)(1-2 t)(1-u t)} .
\end{aligned}
$$

Thus, by using (3) twice, (21) can be written as

$$
\begin{aligned}
& A(u, v)=\frac{\left(u v t^{2}-(t-1)^{2}\right) u v t}{(1-2 t)(1-t)(1-u)} A(u, 1)+\frac{(u v-1)(u v t-v t+2 t-1)}{1-2 t} A(u, 0)+t u A(1, u v) \\
& +\frac{\left(u v t(t-1)+v t(1-2 t)+(t-1)^{2}\right) t v u^{3}}{(1-t)(1-u)(1-2 t)} A(1,1)+\frac{u t(1-u v)(u v t-v t+2 t-1)}{1-2 t} A(1,0) \\
& -\frac{\left(u v(3 u v+2 v-1) t^{3}-\left(\left(u^{2}+5 u+2\right) v^{2}+u v+1\right) t^{2}+\left(2(1+u) v^{2}+(v+1)\right) t-v(1+v)\right) u^{3} v t^{2}}{(1-u t)(1-t)(1-u v t)(1-2 t)} .
\end{aligned}
$$

By a routine computer check, we can verify the following solution for this equation

$$
A(u, v)=\frac{t^{2} u K(u, v)}{(1-u v t)(1-t)^{6}(1-u t)^{2}(1-2 t)^{2}},
$$

where

$$
\begin{aligned}
& K(u, v)=u^{2} v^{2}(1+v)-u^{2} v\left(2(u+5) v^{2}+(u+8) v-1\right) t \\
& +u\left(u\left(u^{2}+19 u+43\right) v^{3}+u(6 u+29) v^{2}+(1-5 u) v+1\right) t^{2} \\
& +\left(1-2 u-u\left(3 u^{2}-6 u+1\right) v+u^{2}(u+3)(u-21) v^{2}-u^{2}\left(9 u^{2}+80 u+104\right) v^{3}\right) t^{3} \\
& +\left((1+u)(1-4 u)+u\left(16 u^{2}-3 u-4\right) v-u^{2}\left(3 u^{2}-39 u-86\right) v^{2}+u^{2}\left(39 u^{2}+192 u+155\right) v^{3}\right) t^{4} \\
& +\left(14 u^{2}+u-5+u\left(2 u^{3}-26 u^{2}+11 u+2\right) v-3 u^{2}(18 u+25) v^{2}-2 u^{2}\left(u^{3}+49 u^{2}+142 u+73\right) v^{3}\right) t^{5} \\
& +\left(2 u^{3}-12 u^{2}+8 u-1-u\left(8 u^{3}-12 u^{2}+7 u-5\right) v-u^{2}\left(2 u^{3}-4 u^{2}-43 u-46\right) v^{2}\right. \\
& \left.+u^{2}\left(10 u^{3}+147 u^{2}+265 u+85\right) v^{3}\right) t^{6} \\
& -u\left(6 u^{2}-2 u-3+\left(1-u^{2}\right)(8 u-1) v-u(u+1)\left(8 u^{2}-7 u-20\right) v^{2}\right. \\
& \left.+u\left(18 u^{3}+133 u^{2}+154 u+28\right) v^{3}\right) t^{7} \\
& +u^{2}\left(4 u-4+\left(2 u^{2}+2 u-3\right) v-\left(10 u^{3}+3 u^{2}-16 u-4\right) v^{2}+\left(14 u^{3}+73 u^{2}+52 u+4\right) v^{3}\right) t^{8} \\
& -4 u^{3} v\left(\left(u^{2}+6 u+2\right) v^{2}+\left(1-u^{2}\right) v+u-1\right) t^{9}+4 u^{4} v^{3} t^{10} .
\end{aligned}
$$

Since $A(1,1)=\sum_{n \geq 2}\left|S_{n}(T)\right| t^{n}$ and so $F_{T}(t)=1+t+A(1,1)$, we get the following result.

ThEOREM 3.5 Let $T=\{1234,1324,3412\}$. Then

$$
F_{T}(x)=\frac{1-9 x+35 x^{2}-75 x^{3}+98 x^{4}-78 x^{5}+34 x^{6}-10 x^{7}}{(1-2 x)^{2}(1-x)^{6}} .
$$




\subsection{Case 72: $\{1243,1324,3412\}$}

First, we look at $G_{2}(x)$. For $\pi=i \pi^{\prime} n \pi^{\prime \prime}$ with 2 left-right maxima and $d \geq 0$ letters in $\pi^{\prime \prime}$, let $H_{d}(x)$ and $J_{d}(x)$ denote the generating functions in the respective cases $i=n-1$ and $i<n-1$. Note $d \geq 1$ in case $i<n-1$. Thus, with $H(x):=\sum_{d \geq 0} H_{d}(x)$ and $J(x):=\sum_{d \geq 1} J_{d}(x)$, we have $G_{2}(x)=H(x)+J(x)$.

LEMMA 3.6

$$
H(x)=\frac{x^{2}\left(1-9 x+34 x^{2}-69 x^{3}+80 x^{4}-54 x^{5}+21 x^{6}-3 x^{7}\right)}{(1-x)^{4}(1-2 x)^{2}\left(1-3 x+x^{2}\right)} .
$$

Proof. Clearly, $H_{0}(x)=x^{2} K(x)=x^{2} F_{\{132,3412\}}(x)$, and so by [26, Seq. A001519], we have $H_{0}(x)=$ $\frac{x^{2}(1-2 x)}{1-3 x+x^{2}}$. For $d \geq 1$, by considering the position of the letter $n-2$ in $\pi^{\prime \prime}$ (either leftmost, rightmost or in the middle), we obtain

$$
H_{d}(x)=x H_{d}(x)+x\left(H_{d}(x)-x^{d+2}\right)+\frac{x^{d+2}}{(1-x)^{d}}+\frac{(K(x)-1) x^{d+4}}{1-x}+\frac{d x^{d+5}}{(1-x)^{2}} .
$$

Note that the last equation also holds for $d=0$. Now sum over $d \geq 0$.

By similar arguments, one can obtain the following result for $J(x)$.

LEMMA 3.7 We have

$$
J(x)=\frac{x^{3}\left(1-4 x+9 x^{2}-11 x^{3}+4 x^{4}-2 x^{5}\right)}{(1-x)^{5}(1-2 x)^{2}} .
$$

Theorem 3.8 Let $T=\{1243,1324,3412\}$. Then

$$
F_{T}(x)=1+\frac{x\left(1-11 x+54 x^{2}-152 x^{3}+268 x^{4}-311 x^{5}+237 x^{6}-109 x^{7}+30 x^{8}-4 x^{9}\right)}{(1-x)^{6}(1-2 x)^{2}\left(1-3 x+x^{2}\right)} .
$$

Proof. Let $G_{m}(x)$ be the generating function for $T$-avoiders with $m$ left-right maxima. Clearly, $G_{0}(x)=1$ and $G_{1}(x)=x F_{T}(x)$. By Lemmas 3.6 and 3.7, we have

$$
G_{2}(x)=H(x)+J(x)=\frac{x^{2}\left(1-9 x+36 x^{2}-81 x^{3}+107 x^{4}-88 x^{5}+50 x^{6}-14 x^{7}+x^{8}\right)}{(1-x)^{5}(1-2 x)^{2}\left(1-3 x+x^{2}\right)} .
$$

Now, let us write an equation for $G_{m}(x)$ where $m \geq 3$. Let $\pi=i_{1} \pi^{(1)} \cdots i_{m} \pi^{(m)} \in S_{n}(T)$ with exactly $m$ left-right maxima. Since $\pi$ avoids $1324, \pi^{(s)}<i_{1}$ for all $s=1,2, \ldots, m-1$ and, since $\pi$ avoids $1243, \pi^{(m)}<i_{2}$. If $\pi^{(m-1)}=\emptyset$, then we have a contribution of $x G_{m-1}(x)$. Otherwise, since $m \geq 3$ and $\pi$ avoids 3412, we see that $\pi^{(m)}<i_{1}$. Moreover, since $\pi$ avoids 3412 and 1324 , we see that $\pi^{(1)}>\pi^{(2)}>\cdots>\pi^{(m)}$ and $\pi^{(2)} \cdots \pi^{(m)}$ is decreasing, where $\pi^{(m-1)}$ is not empty and $\pi^{(1)}$ avoids 132 and 3412. Therefore, we have a contribution of $x^{m+1} K(x) /(1-x)^{m-1}$, where $K(x)$ is given in the proof of Lemma 3.6. Hence,

$$
G_{m}(x)=x G_{m-1}(x)+\frac{x^{m+1}}{(1-x)^{m-1}} \frac{1-2 x}{1-3 x+x^{2}} .
$$


Summing over all $m \geq 3$ and using the expressions for $G_{0}(x)$ and $G_{1}(x)$, we get

$$
F_{T}(x)-1-x F_{T}(x)-G_{2}(x)=x\left(F_{T}(x)-1-x F_{T}(x)\right)+\frac{x^{4}}{(1-x)\left(1-3 x+x^{2}\right)} .
$$

Solve for $F_{T}(x)$ using (4) to complete the proof.

3.4 Case 76: $\{3412,1324,2341\}$

Here we use the fact that

$$
F_{\{132,2341,3412\}}(x)=F_{\{213,2341,3412\}}(x)=\frac{1-3 x+x^{2}}{(1-x)^{2}(1-2 x)},
$$

denoted $A$ for short (this follows from the main result in [24]).

Lemma 3.9 The generating function $G_{2}(x)$ for T-avoiders with two left-right maxima is given by

$$
G_{2}(x)=\frac{x^{2}\left(1-7 x+23 x^{2}-40 x^{3}+39 x^{4}-22 x^{5}+9 x^{6}-4 x^{7}\right)}{(1-x)^{6}(1-2 x)^{2}} .
$$

Proof. Suppose $\pi=i \alpha n \beta \in S_{n}(T)$ with two left-right maxima. If $i=1$, then $\pi=1 n \beta$ where $\beta$ avoids $\{213,2341,3412\}$, giving a contribution to $G_{2}(x)$ of $x^{2} A$. Now suppose $i>1$. If $i-1$ is the leftmost letter of $\alpha$, we obtain a contribution of $x G_{2}(x)$ by deleting $i-1$. If $i-1 \in \alpha$ and is not the leftmost letter, then $\pi$ decomposes as $\pi=i \alpha^{\prime}(i-1) \alpha^{\prime \prime} n \beta^{\prime} \beta^{\prime \prime}$, where $\beta^{\prime}>i>i-1>\beta^{\prime \prime}>\alpha^{\prime}>\alpha^{\prime \prime}$ (to avoid 1324) and $\beta^{\prime \prime}$ is decreasing (to avoid 3412), as in Figure 1. Consider now four cases according as to

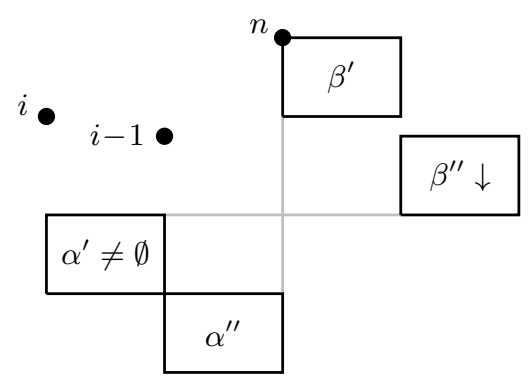

Figure 1: An avoider with 2 left-right maxima, $i-1$ before $n$ but not adjacent to $i$

whether $\alpha^{\prime \prime}$ or $\beta^{\prime \prime}$ is empty or not:

- $\alpha^{\prime \prime}, \beta^{\prime \prime}$ both empty. Note that $\alpha^{\prime}$ is nonempty and avoids $\{132,2341,3412\}$ and $\beta^{\prime}$ avoids $\{213,2341,3412\}$. So the contribution is $x^{3}(A-1) A$.

- $\alpha^{\prime \prime}=\emptyset, \beta^{\prime \prime} \neq \emptyset$. Here, $\beta^{\prime}$ is decreasing (to avoid 2341), while $\alpha^{\prime}$ is nonempty and avoids $\{132,2341,3412\}$. So the contribution is $x^{4}(A-1) /(1-x)^{2}$.

$-\alpha^{\prime \prime} \neq \emptyset, \beta^{\prime \prime}=\emptyset$. Here, $\alpha^{\prime}$ is decreasing (to avoid 2341) and nonempty, $\alpha^{\prime \prime}$ is also decreasing (to avoid 3412$)$ and nonempty, and $\beta^{\prime}$ avoids $\{213,2341,3412\}$. So the contribution is $x^{5} A /(1-x)^{2}$. 
- $\alpha^{\prime \prime} \neq \emptyset, \beta^{\prime \prime} \neq \emptyset$. Here, $\alpha^{\prime}$ and $\beta^{\prime}$ are decreasing (both to avoid 2341) and $\alpha^{\prime}$ is nonempty, $\alpha^{\prime \prime}$ is also decreasing (to avoid 3412) and nonempty, and $\beta^{\prime \prime}$ is nonempty (and decreasing, as always). So the contribution is $x^{6} /(1-x)^{4}$.

Next, suppose $i>1$ and $i-1 \in \beta$ so that $\pi=i \alpha n \beta^{\prime}(i-1) \beta^{\prime \prime}$. Then $\beta^{\prime}>i$ since $j \in \beta^{\prime}$ with $j<i$ makes $\operatorname{inj}(i-1)$ a 3412. For $d \geq 0$, let $B_{d}$ be the generating function for such permutations where $\beta^{\prime}$ has $d$ letters.

LEMMA 3.10 We have

$$
B_{0}=\frac{x^{3}\left(1-5 x+12 x^{2}-13 x^{3}+4 x^{4}+4 x^{5}-4 x^{6}\right)}{(1-x)^{4}(1-2 x)^{2}} .
$$

Proof. First, let $K$ be the generating function for $T$-avoiders $i(i-2)(i-4) \pi^{\prime}(i+1)(i-1)(i-3) \pi^{\prime \prime}$ with two left-right maxima such that $i \geq 5$ where $n=i+1$. Let us write an equation for $K$. When $i=5$, the contribution is $x^{6}$. Otherwise, we consider the position of $i-5$. If $i-5$ is either the leftmost letter of $\pi^{\prime}$ or of $\pi^{\prime \prime}$, then we have a contribution of $x K$. Otherwise, $\pi^{\prime}=\alpha^{\prime}(i-5) \alpha^{\prime \prime}$ with $\alpha^{\prime} \neq \emptyset$ such that $i-5>\pi^{\prime \prime}>\alpha^{\prime}>\alpha^{\prime \prime}$ where $\alpha^{\prime \prime}$ and $\pi^{\prime \prime}$ are decreasing. Upon considering whether $\alpha^{\prime \prime}$ is empty or not, we obtain the contribution $x^{7}\left(A-1+x^{2} /(1-x)^{2}\right) /(1-x)$. Thus

$$
K=2 x K+x^{6}+x^{7}\left(A-1+x^{2} /(1-x)^{2}\right) /(1-x),
$$

which leads to

$$
K=\frac{\left(1-4 x+6 x^{2}-2 x^{3}\right) x^{6}}{(1-2 x)^{2}(1-x)^{2}} .
$$

Next, let $K^{\prime}$ be the generating function for $T$-avoiders $i(i-2) \pi^{\prime} n(i-1)(i-3) \pi^{\prime \prime}$ with two left-right maxima such that $n>i \geq 4$. Let us write an equation for $K^{\prime}$. When $i=4$, we have a contribution of $x^{5} A$. If $i-4$ is the leftmost letter of $\pi^{\prime}$, then we have a contribution $K$, and if $i-4$ is leftmost letter of $\pi^{\prime \prime}$, then we have a contribution $x K^{\prime}$. Otherwise, $\pi^{\prime}=\alpha^{\prime}(i-4) \alpha^{\prime \prime}$ with $\alpha^{\prime} \neq \emptyset$ such that $i-4>$ $\pi^{\prime \prime}>\alpha^{\prime}>\alpha^{\prime \prime}$ with $\alpha^{\prime \prime}$ and $\pi^{\prime \prime}$ decreasing. By considering whether $\alpha^{\prime \prime}$ is empty or not, we obtain the contribution $x^{6}\left(A-1+x^{2} /(1-x)^{2}\right) /(1-x)$. Thus $K^{\prime}=x^{5} A+x K^{\prime}+K+x^{6}\left(A-1+x^{2} /(1-x)^{2}\right) /(1-x)$, which leads to

$$
K^{\prime}=\frac{\left(1-4 x+6 x^{2}-2 x^{3}-2 x^{4}\right) x^{5}}{(1-2 x)^{2}(1-x)^{3}} .
$$

Next, let $K^{\prime \prime}$ be the generating function for $T$-avoiders $i(i-2) \pi^{\prime} n(i-1) \pi^{\prime \prime}$ with 2 left-right maxima such that $n>i \geq 3$. By using similar techniques as for finding formulas $K$ and $K^{\prime}$, we have

$$
K^{\prime \prime}=x^{4} A+x K^{\prime \prime}+K^{\prime}+x^{5} A(A-1)+\frac{x^{7} A}{(1-x)^{2}}+\frac{x^{6}(A-1)}{1-x}+\frac{x^{8}}{(1-x)^{3}},
$$

which implies

$$
K^{\prime \prime}=\frac{\left(1-3 x+4 x^{2}\right) x^{4}}{(1-2 x)^{2}(1-x)^{2}} .
$$

Lastly, let $K^{\prime \prime \prime}$ be the generating function for $T$-avoiders $i \pi^{\prime} n(i-1) j(i-2) \pi^{\prime \prime}$ with two left-right maxima such that $n>j>i \geq 3$. By a similar method, we obtain

$$
K^{\prime \prime \prime}=\frac{\left(1-x+2 x^{3}\right) x^{5}}{(1-2 x)(1-x)^{2}} .
$$


Now, we are ready to write an equation for the generating function $B_{0}$ for $T$-avoiders $i \pi^{\prime} n(i-1) \pi^{\prime \prime}$ containing two left-right maxima such that $n>i \geq 2$. Again, we decompose the structure by considering the position of $i-2$. If $i=2$, then we have a contribution of $x^{3} A$, if $i-2$ is leftmost letter of $\pi^{\prime}$, then we have a contribution of $K^{\prime \prime}$, if $i-2$ belongs to $\pi^{\prime \prime}$ and is not the leftmost letter of it, we have a contribution of $x^{4} A(A-1)+\frac{x^{6} A}{(1-x)^{2}}+\frac{x^{5}(A-1)}{1-x}+\frac{x^{7}}{(1-x)^{3}}$, and if $i-2$ belongs to $\pi^{\prime \prime}$, we have a contribution of $K^{\prime \prime \prime}$. Thus,

$$
B_{0}=x^{3} A+K^{\prime \prime}+x^{4} A(A-1)+\frac{x^{6} A}{(1-x)^{2}}+\frac{x^{5}(A-1)}{1-x}+\frac{x^{7}}{(1-x)^{3}}+K^{\prime \prime \prime},
$$

which completes the proof.

One can similarly show (details omitted) that

$$
B_{1}=\frac{x^{4}\left(1-2 x+2 x^{2}\right)\left(1-2 x+2 x^{2}-2 x^{4}\right)}{(1-x)^{4}(1-2 x)^{2}},
$$

and $B_{d}=x B_{d-1}$ for all $d \geq 2$. Hence, the total contribution for the case $i>1$ and $i-1 \in \beta$ is given by $B$, where $B-B_{1}-B_{0}=x\left(B-B_{0}\right)$, which leads to

$$
B=\frac{x^{3}\left(1-5 x+13 x^{2}-17 x^{3}+9 x^{4}+2 x^{5}-4 x^{6}\right)}{(1-x)^{5}(1-2 x)^{2}} .
$$

Adding all the contributions,

$$
G_{2}(x)=x^{2} A+x G_{2}(x)+x^{3}(A-1) A+\frac{x^{4}(A-1)}{(1-x)^{2}}+\frac{x^{5} A}{(1-x)^{2}}+\frac{x^{6}}{(1-x)^{4}}+B,
$$

with solution for $G_{2}(x)$ as claimed.

Theorem 3.11 Let $T=\{3412,1324,2341\}$. Then

$$
F_{T}(x)=\frac{1-10 x+44 x^{2}-110 x^{3}+173 x^{4}-176 x^{5}+114 x^{6}-45 x^{7}+12 x^{8}-4 x^{9}}{(1-x)^{7}(1-2 x)^{2}} .
$$

Proof. Let $G_{m}(x)$ be the generating function for $T$-avoiders with $m$ left-right maxima. Clearly, $G_{0}(x)=1, G_{1}(x)=x F_{T}(x)$, with $G_{2}(x)$ as given above.

Now, let us write an equation for $G_{m}(x)$ where $m \geq 3$. Let $\pi=i_{1} \pi^{(1)} \cdots i_{m} \pi^{(m)} \in S_{n}(T)$ with exactly $m$ left-right maxima. Since $\pi$ avoids 1324 and 2341, we can write $\pi$ as

$$
\pi=i_{1} \pi^{(1)} i_{2} \pi^{(2)} i_{3} i_{4} \cdots i_{m} \gamma \beta
$$

where $\pi^{(1)}>\pi^{(2)}$ and $\gamma>i_{m-1}>\beta>i_{m-2}$. By considering the four possibilities based on whether $\pi^{(2)}$ or $\beta$ is empty or not, we obtain the contributions $x^{m} A^{2}$ (both empty), $\frac{x^{m+1}}{(1-x)^{2}} A\left(\pi^{(2)}\right.$ empty, $\beta$ nonempty), $\frac{x^{m+1}}{(1-x)^{2}} A\left(\pi^{(2)}\right.$ nonempty, $\beta$ empty) and $\frac{x^{m+2}}{(1-x)^{4}}$ (both nonempty). Thus,

$$
G_{m}(x)=x^{m}\left(A+\frac{x}{(1-x)^{2}}\right)^{2} .
$$


Therefore,

$$
F_{T}(x)-1-x F_{T}(x)-G_{2}(x)=\sum_{m \geq 3} G_{m}(x)=\frac{x^{3}}{1-x}\left(A+\frac{x}{(1-x)^{2}}\right)^{2} .
$$

Substituting for $G_{2}(x)$ and solving for $F_{T}(x)$ completes the proof.

\subsection{Case 88: $\{3412,3421,1324\}$}

Our proof in this case is based on a labelled generating forest. The following lemma both recursively defines labels and provides valid succession rules. The $j$-th entry on the right-hand side in the rules gives the label when $n+1$ is inserted into the $j$-th active site left to right. A label $k^{i}, 1 \leq i \leq 5$, always indicates $k$ active sites. The proof is omitted.

LEMMA 3.12 The generating forest $\mathcal{F}$ is given by

$$
\begin{array}{ll}
\text { Roots: } & 3^{1}, 3^{3} \\
\text { Rules: } & k^{1} \rightsquigarrow 3^{1} 2^{5} 3^{5} \cdots(k-2)^{5}(k+1)^{1}(k+1)^{2}, \quad k \geq 3, \\
& k^{2} \rightsquigarrow 3^{3} 2^{5} 3^{5} \cdots(k-3)^{5}(k-1)^{4} k^{4}(k+1)^{3}, \quad k \geq 4, \\
& k^{3} \rightsquigarrow 3^{3} 2^{5} 3^{5} \cdots(k-2)^{5} k^{4}(k+1)^{3}, \quad k \geq 3, \\
& 2^{3} \rightsquigarrow 2^{3} 3^{4}, \\
& k^{4} \rightsquigarrow 2^{3} 2^{5} 3^{5} \cdots(k-1)^{5}(k+1)^{4}, \quad k \geq 3, \\
& k^{5} \rightsquigarrow 1^{5} 2^{5} 3^{5} \cdots k^{5}, \quad k \geq 1 .
\end{array}
$$

Theorem 3.13 Let $T=\{3412,3421,1324\}$. Then

$$
F_{T}(x)=\frac{(1-x)^{2}\left(1-5 x+7 x^{2}+x^{3}\right)}{(1-2 x)^{4}} .
$$

Proof. Let $a_{k}(x), b_{k}(x), c_{k}(x), d_{k}(x)$ and $e_{k}(x)$ be the generating functions for the number of permutations in the $n$th level of the generating forest $\mathcal{F}$ with label $k^{1}, k^{2}, k^{3}, k^{4}$ and $k^{5}$, respectively. By Lemma 3.12, we have

$$
\begin{aligned}
& a_{k}(x)=x a_{k-1}(x), \quad k \geq 4, \\
& b_{k}(x)=x a_{k-1}(x), \quad k \geq 4, \\
& c_{k}(x)=x\left(b_{k-1}+c_{k-1}(x)\right), \quad k \geq 4, \\
& d_{k}(x)=x\left(d_{k-1}(x)+c_{k}(x)+b_{k}(x)+b_{k+1}(x)\right), \quad k \geq 4, \\
& e_{k}(x)=x e_{k}(x)+x \sum_{j \geq k+1}\left(d_{j}(x)+e_{j}(x)\right)+x \sum_{j \geq k+2}\left(a_{j}(x)+c_{j}(x)\right)+x \sum_{j \geq k+3} b_{j}(x), \quad k \geq 2,
\end{aligned}
$$

with $a_{3}(x)=x^{2}+x \sum_{j \geq 3} a_{j}(x), c_{2}(x)=x c_{2}(x)+x \sum_{j>3} d_{j}(x), c_{3}(x)=x^{2}+x c_{3}(x)+x \sum_{j \geq 4}\left(b_{j}(x)+\right.$ $\left.c_{j}(x)\right), d_{3}(x)=x c_{2}(x)+x c_{3}(x)+x b_{4}(x)$ and $e_{1}(x)=x \sum_{j \geq 1} e_{j}(x)$.

Now let $A(x, v)=\sum_{k \geq 3} a_{k}(x) v^{k}, B(x, v)=\sum_{k \geq 3} b_{k}(x) v^{k}, C(x, v)=\sum_{k \geq 3} c_{k}(x) v^{k}, D(x, v)=$ $\sum_{k \geq 2} d_{k}(x) v^{k}$ and $E(x, v)=\sum_{k \geq 2} e_{k}(x) v^{k}$. 
By the above recurrences, we see that $A(x, v)-\left(x^{2}+x A(x, 1)\right) v^{3}=x v A(x, v)$. Thus, by substituting $v=1$, we obtain $A(x, 1)=\frac{x^{2}}{1-2 x}$, which implies $A(x, v)=\frac{x^{2} v^{3}(1-x)}{(1-x v)(1-2 x)}$. Thus, by the equation for $b_{k}(x)$, we have $B(x, v)=\frac{x^{3} v^{4}(1-x)}{(1-x v)(1-2 x)}$.

By the equations for $c_{k}(x), d_{k}(x), e_{k}(x)$, we have

$$
\begin{aligned}
& C(x, v)-c_{3}(x) v^{3}-c_{2}(x) v^{2}=x v\left(B(x, v)+C(x, v)-c_{2}(x) v^{2}\right), \\
& D(x, v)-d_{3}(x)=x\left(v D(x, v)+C(x, v)-c_{3}(x) v^{3}-c_{2}(x) v^{2}+B(x, v)+\left(B(x, v)-b_{4}(x) v^{4}\right) / v\right), \\
& E(x, v)-e_{1}(x) v=\frac{x}{1-v}\left(v^{2} E(x, 1)-v E(x, v)+v^{2} D(x, 1)-D(x, v)+v^{3} C(x, 1)-C(x, v)\right) \\
& +x v^{2} c_{2}(x)+\frac{x}{1-v}\left(v^{4} B(x, 1)-B(x, v)+v^{3} A(x, 1)-A(x, v)\right),
\end{aligned}
$$

with $c_{2}(x)=\frac{x}{1-x} D(x, 1), c_{3}(x)=x^{2}+x B(x, 1)+x C(x, 1)-\frac{x^{2}}{1-x} D(x, 1), d_{3}(x)=\frac{x^{2}}{(1-x)^{2}} D(x, 1)$ and $e_{1}(x)=x E(x, 1) v$. Note that $b_{4}(x)=\frac{x^{3}(1-x)}{1-2 x}($ from $B(x, v))$.

Substituting $v=1$ in the first two equations, and solving for $C(x, 1)$ and $D(x, 1)$, we obtain

$$
C(x, 1)=\frac{x^{2}\left(1-4 x+7 x^{2}-4 x^{3}-2 x^{4}\right)}{(1-2 x)^{3}}, \quad D(x, 1)=\frac{x^{3}(1-x)\left(1-2 x^{2}\right)}{(1-2 x)^{3}},
$$

which implies

$$
\begin{aligned}
C(x, v) & =\frac{x^{2} v^{2}\left(x^{2}\left(1-2 x^{2}\right)+\left(1-5 x+4 x^{5}+9 x^{2}-8 x^{3}\right) v\right)}{(1-x v)^{2}(1-2 x)^{3}}, \\
& +\frac{x^{3}(1-x) v^{4}\left(\left(x^{2}+2 x-1\right)\left(2 x^{2}-2 x+1\right)+x(1-2 x)^{2} v\right)}{(1-x v)^{2}(1-2 x)^{3}}, \\
D(x, v) & =\frac{x^{3} v^{3}\left(1-4 x+5 x^{2}+2 x^{3}-6 x^{4}+x^{2}\left(8 x^{3}-6 x^{2}+2 x-1\right) v+x^{4}\left(1-2 x^{2}\right) v^{2}\right)}{(1-x v)^{3}(1-2 x)^{3}} .
\end{aligned}
$$

We solve the equation for $E(x, v)$ by using the kernel method (see, e.g., [16] for an exposition), taking $v=\frac{1}{1-x}$. Using the expressions for $A(x, v), B(x, v), C(x, v)$ and $D(x, v)$, this gives

$$
E(x, 1)=\frac{x^{4}(x+1)(3-4 x)}{(1-2 x)^{4}} .
$$

Since $F_{T}(x)=1+x+A(x, 1)+B(x, 1)+C(x, 1)+D(x, 1)+E(x, 1)$, the result follows.

3.6 Case 156: $T=\{1324,2341,2431\}$

Theorem 3.14 Let $T=\{1324,2341,2431\}$. Then

$$
F_{T}(x)=\frac{1-8 x+23 x^{2}-25 x^{3}+3 x^{4}+7 x^{5}}{(1-2 x)^{2}\left(1-3 x+x^{2}\right)\left(1-2 x-x^{2}\right)} .
$$

Proof. Let $G_{m}(x)$ be the generating function for $T$-avoiders with $m$ left-right maxima. Clearly, $G_{0}(x)=1$ and $G_{1}(x)=x F_{T}(x)$. 
Let us write an equation for $G_{2}(x)$. Let $\pi=i \pi^{\prime} n \pi^{\prime \prime} \in S_{n}(T)$ with exactly two left-right maxima. The contributions for the cases $n-2 \geq i=1$ and $i=n-1 \geq 1$ are $x^{2}(M(x)-1)$ and $x\left(F_{T}(x)-1\right)$, respectively, where $M(x)=\frac{1-x-x^{2}}{1-2 x-x^{2}}$ is the generating function for $\{213,2341,2431\}$-avoiders (for example, see [24]). Denote the contribution for the case $2 \leq i \leq n-2$ by $H(x)$. Then $G_{2}(x)=$ $x^{2}(M(x)-1)+x\left(F_{T}(x)-1\right)+H(x)$. To find a formula for $H(x)$, we consider the position of $i-1$ in $\pi$, which leads to either $\pi=i \alpha(i-1) \beta^{\prime} n \beta^{\prime \prime} \beta^{\prime \prime \prime}$ with $\beta^{\prime \prime \prime}>i \alpha>\beta^{\prime} \beta^{\prime \prime}$ or $\pi=i \alpha^{\prime} n \alpha^{\prime \prime}(i-1) \beta^{\prime} \beta^{\prime \prime}$ with $\beta^{\prime \prime}>i>\alpha^{\prime} \alpha^{\prime \prime}>\beta^{\prime}$. By examining the four possibilities, $\alpha, \beta^{\prime} \beta^{\prime \prime}$ either empty or not, in the first case and examining the four possibilities for $\alpha^{\prime} \alpha^{\prime \prime}, \beta^{\prime}$ in the second case, we obtain

$$
\begin{aligned}
H(x) & =x^{3}(M(x)-1)+x^{3}(K(x)-1)(M(x)-1)+\frac{x^{4}}{1-x}(K(x)-1)(M(x)-1) \\
& +x^{3}(M(x)-1)+x^{3}(K(x)-1)(M(x)-1)+\frac{x^{4}}{1-x}(K(x)-1)(M(x)-1)+2 x H(x),
\end{aligned}
$$

where $K(x)=\frac{1-2 x}{1-3 x+x^{2}}$ is the generating function for $\{132,2341\}$-avoiders. Thus,

$$
H(x)=\frac{2 x^{4}(1-x)^{2}}{(1-2 x)\left(1-3 x+x^{2}\right)\left(1-2 x-x^{2}\right)} .
$$

Next, let us write an equation for $G_{3}(x)$. Let $\pi=i_{1} \pi^{\prime} i_{2} \pi^{\prime \prime} i_{3} \pi^{\prime \prime \prime} \in S_{n}(T)$ with exactly 3 left-right maxima. Since $\pi$ avoids $T$, we can write $\pi=i_{1} \pi^{\prime} i_{2} \pi^{\prime \prime} i_{3} \beta \alpha$ where $\pi^{\prime \prime}<\pi^{\prime}<i_{1}<\beta<i_{2}<\alpha<i_{3}$. By considering the four cases based on whether $\pi^{\prime \prime}$ or $\beta$ is empty or not, we obtain

$$
G_{3}(x)=x^{3}\left(K(x) M(x)+K(x)(M(x)-1)+\frac{(K(x)-1) M(x)}{1-x}+\frac{(K(x)-1)(M(x)-1)}{1-x}\right) .
$$

Finally, let us write an equation for $G_{m}(x)$ with $m \geq 4$. Let $\pi=i_{1} \pi^{(1)} \cdots i_{m} \pi^{(m)} \in S_{n}(T)$ with exactly $m$ left-right maxima. Since $\pi$ avoids $T$, we see that $i_{1}>\pi^{(1)}>\pi^{(2)}, \pi^{(j)}=\emptyset$ for $j=3,4, \ldots, m-1$, and $\pi^{(m)}$ has the form $\beta \alpha$ where $i_{m}>\alpha>i_{m-1}>\beta$. Thus, $\pi$ avoids $T$ if and only if the permutation that is obtained from $\pi$ by removing $i_{m-2}$ avoids $T$. Hence $G_{m}(x)=x G_{m-1}(x)=$ $\cdots=x^{m-3} G_{3}(x)$ for $m \geq 3$.

By summing over $m \geq 4$ and using the expressions for $G_{0}(x), G_{1}(x), G_{2}(x)$ and $G_{3}(x)$, we obtain

$$
\begin{aligned}
& F_{T}(x)=1+x F_{T}(x)+x\left(F_{T}(x)-1\right)+x^{2}(M(x)-1)+H(x) \\
& +\frac{x^{3}}{1-x}\left(K(x) M(x)+K(x)(M(x)-1)+\frac{(K(x)-1) M(x)}{1-x}+\frac{(K(x)-1)(M(x)-1)}{1-x}\right),
\end{aligned}
$$

which, by solving for $F_{T}(x)$, completes the proof.

\subsection{Case 158: $\{1324,1342,3412\}$}

Here, it is convenient to consider $J(x)$, the generating function for $T$-avoiders whose maximal letter occurs in second position, and its refinement to $J_{d}(x)$, the generating function for $T$-avoiders whose maximal letter occurs in second position and whose first letter is $d$, that is, permutations of the form $d n \pi^{\prime \prime} \in S_{n}(T)$. 
LEMMA 3.15 With the preceding notation,

$$
J_{1}(x)=\frac{x^{2}(1-x)}{1-2 x}, \quad J_{2}(x)=\frac{x^{3}\left(1-2 x+2 x^{2}\right)}{(1-2 x)^{2}}, \quad J(x)=\frac{x^{2}(1-2 x)}{(1-x)(1-3 x)} .
$$

Proof. Suppose $\pi \in S_{n}(T)$ has the form $d n \pi^{\prime \prime}$. Since $\pi$ avoids 3412 , we see that $\pi^{\prime \prime}$ contains the subsequence $(d-1)(d-2) \cdots 1$. Therefore, if $d=n-1$, the contribution is $x^{d+1}$ and otherwise, by considering whether $n-1$ occurs before or after the string $(d-1)(d-2) \cdots 1$, we find

$$
J_{d}(x)=x^{d+1}+\sum_{j=1}^{d} x^{j} J_{d+1-j}+\sum_{j=1}^{d} x^{j}\left(J_{d+1-j}-x^{d+2-j}\right),
$$

for all $d \geq 1$. Solving this recurrence successively for $d=1$ and $d=2$ gives the first two stated expressions. Also, summing over all $d \geq 1$ yields

$$
J(x)=\frac{x^{2}}{1-x}+\frac{2 x}{1-x} J(x)-\frac{x^{3}}{(1-x)^{2}},
$$

from which the expression for $J(x)$ follows.

LEMmA 3.16 Let $G_{2}(x)$ be the generating function for the number of T-avoiders with exactly two left-right maxima. Then $G_{2}(x)=\frac{x^{2}\left(1-7 x+19 x^{2}-23 x^{3}+9 x^{4}\right)}{(1-x)^{2}(1-2 x)(1-3 x)\left(1-3 x+x^{2}\right)}$.

Proof. Again, we refine $G_{2}(x)$ by defining $G_{2}(x ; d)$ to be the generating function for the number of permutations $i \pi^{\prime} n \pi^{\prime \prime} \in S_{n}(T)$ with exactly two left-right maxima where $\pi^{\prime \prime}$ has $d$ letters smaller than $i$. Since $\pi$ avoids 3412 , we see that $\pi^{\prime \prime}$ is decreasing. Now, let us write an equation for $G_{2}(x ; d)$. Clearly, $G_{2}(x ; 0)=x^{2} F_{\{132,3412\}}(x) F_{\{213,231\}}(x)=\frac{x^{2}(1-x)}{1-2 x} K(x)$, where $F_{\{132,3412\}}(x)=K(x)=\frac{1-2 x}{1-3 x+x^{2}}$ (see [26, Seq A001519]) and $F_{\{213,231\}}(x)=L(x)=\frac{1-x}{1-2 x}$.

For $d=1$, let $j$ denote the letter in $\pi^{\prime \prime}$ smaller than $i$. Thus $i>2$ and $\pi$ can be written, according as $j>1$ or $j=1$, as either $(1) \pi=i(i-1) \cdots(j+1) \alpha^{(1)} n(n-1) \cdots\left(i^{\prime}+1\right) i^{\prime} j \beta^{(1)}$, where $1 \in \alpha^{(1)}$ and $j>\alpha^{(1)}, i^{\prime}>\beta^{(1)}$ and $\beta^{(1)}$ avoids 213 and 231 and $\alpha^{(1)}$ avoids 132 and 3412, or (2) $\pi=i \alpha n \beta$, where $\alpha$ consists of the letters $2,3, \ldots, i-1$ in some order and $\alpha$ avoids 132 and 3412 . Hence,

$$
G_{2}(x ; 1)=\frac{x^{3}}{(1-x)(1-2 x)}(K(x)-1)+K(x) J_{2}(x),
$$

where $J_{2}(x)$ is defined above.

Now, let $d \geq 2$. Since $\pi$ avoids 1324 and 1342, we can express $\pi$ as

$$
\pi=i \alpha^{(1)} \alpha^{(2)} \cdots \alpha^{(d+1)} n \beta^{(1)} j_{1} \cdots \beta^{(d)} j_{d} \beta^{(d+1)},
$$

where $i>\alpha^{(d+1)}>j_{1}>\alpha^{(d)}>j_{2}>\cdots>\alpha^{(2)}>j_{d}>\alpha^{(1)}$. We consider three cases:

- $\alpha^{(s)} \neq \emptyset$ and $\alpha^{(s+1)}=\cdots=\alpha^{(d+1)}=\emptyset$ with $s=3,4, \ldots, d+1$. In this case $\alpha^{(1)}, \ldots, \alpha^{(s-1)}$ are decreasing and $\alpha^{(s)}$ avoids 132 and 3412, while $\beta^{(2)}=\cdots=\beta^{(d+1)}=\emptyset$ and $\beta^{(1)}$ is decreasing. Hence, we have a contribution of $\frac{x^{d+2}}{(1-x)^{s}}(K(x)-1)$. 
- $\alpha^{(3)}=\cdots=\alpha^{(d+1)}=\emptyset$ and $\alpha^{(2)} \neq \emptyset$. In this case, $\alpha^{(1)}$ and $\beta^{(1)}$ are decreasing, and $\beta^{(1)}>$ $\beta^{(2)}>\cdots>\beta^{(d+1)}$. Thus, we have a contribution of $\frac{x}{(1-x)^{2}}(K(x)-1) J_{d}(x)$.

- $\alpha^{(2)}=\cdots=\alpha^{(d+1)}=\emptyset$. In this case, we have a contribution of $K(x) J_{d+1}(x)$.

Hence, for all $d \geq 2$,

$$
G_{2}(x ; d)=\sum_{s=3}^{d+1} \frac{x^{d+2}}{(1-x)^{s}}(K(x)-1)+\frac{x}{(1-x)^{2}}(K(x)-1) J_{d}(x)+K(x) J_{d+1}(x) .
$$

Summing over $d \geq 0$ and using Lemma 3.15, we obtain the stated expression for $G_{2}(x)$.

Theorem 3.17 Let $T=\{1324,1342,3412\}$. Then

$$
F_{T}(x)=\frac{1-10 x+40 x^{2}-81 x^{3}+88 x^{4}-50 x^{5}+11 x^{6}}{(1-x)^{3}(1-2 x)(1-3 x)\left(1-3 x+x^{2}\right)} .
$$

Proof. Let $G_{m}(x)$ be the generating function for $T$-avoiders with $m$ left-right maxima. Clearly, $G_{0}(x)=1$ and $G_{1}(x)=x F_{T}(x)$, with $G_{2}(x)$ given by Lemma 3.16.

Now, let us write an equation for $G_{m}(x)$ where $m \geq 3$. A $T$-avoider $i_{1} \pi^{(1)} \cdots i_{m} \pi^{(m)}$ with $m \geq 3$ left-right maxima $i_{1}, i_{2}, \ldots, i_{m}$ has the restricted form shown in Figure 2, where $i_{1}, i_{2}, \ldots, i_{m-1}$ are

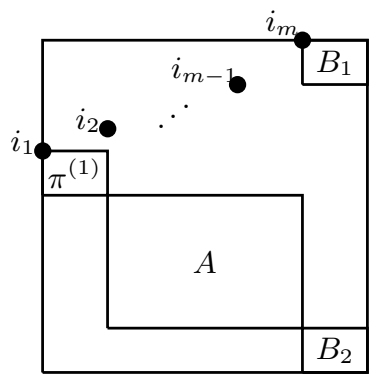

Figure 2: A $\{1324,1342,3412\}$-avoider with $m \geq 3$ left-right maxima

increasing consecutive integers, $A$ is a list of zero or more decreasing consecutive integers, $\pi^{(m)}$ is composed of $B_{1}$ and $B_{2}$, and regions not marked are empty. Furthermore, $\pi^{(1)}$ avoids $\{132,3412\}$ and, of course, $i_{1} i_{m} \pi^{(m)}$ avoids $T$. Conversely, every permutation of this form with $m \geq 3$ satisfying the latter two conditions is a $T$-avoider. Hence, we get contributions as follows: $K(x)$ from $\pi^{(1)} ; J(x)$ from $i_{1} i_{m} \pi^{(m)} ; x^{m-2}$ from $i_{2}, \ldots, i_{m-1}$; and $1 /(1-x)^{m-2}$ from $A$. So $G_{m}(x)=x^{m-2} K(x) J(x) /(1-x)^{m-2}$. By summing over $m \geq 3$, we obtain

$$
F_{T}(x)-1-x F_{T}(x)-G_{2}(x)=\frac{x^{3}(1-2 x)}{(1-x)(1-3 x)\left(1-3 x+x^{2}\right)} .
$$

Now solve for $F_{T}(x)$ using Lemma 3.16 to complete the proof. 
3.8 Case 180: $\{1342,2314,4231\}$

Theorem 3.18 Let $T=\{1342,2314,4231\}$. Then

$$
F_{T}(x)=\frac{1-7 x+18 x^{2}-22 x^{3}+16 x^{4}-6 x^{5}+x^{6}-\left(x-5 x^{2}+8 x^{3}-2 x^{4}-2 x^{5}+x^{6}\right) C(x)}{(1-2 x)(1-x)^{2}\left(1-5 x+4 x^{2}-x^{3}\right)} .
$$

Proof. Let $G_{m}(x)$ be the generating function for $T$-avoiders with $m$ left-right maxima. Clearly, $G_{0}(x)=1$ and $G_{1}(x)=x F_{\{231\}}(x)=x C(x)$ (see [17]). Now let us write an equation for $G_{m}(x)$ for $m \geq 3$. Let $\pi=i_{1} \pi^{(1)} i_{2} \pi^{(2)} \cdots i_{m} \pi^{(m)} \in S_{n}(T)$ with exactly $m$ left-right maxima. Since $\pi$ avoids 2314 and 1342, we see that $\pi^{(j)}>i_{j-1}$ for all $j=2,3, \ldots, m-1$. If $\pi^{(m)}$ has a letter smaller than any letter in $\pi^{(1)}$, then $i_{j} \pi^{(j)}=i_{j}\left(i_{j}-1\right) \cdots\left(i_{j-1}+1\right)$ for all $j=1,2, \ldots, m-1$, and $\pi^{(m)}=i_{m}\left(i_{m}-1\right) \cdots\left(i_{m-1}+1\right) \beta$ such that $\beta$ is a nonempty permutation in $S_{i_{0}}(231)$. Hence, we get a contribution of $\frac{x^{m}}{(1-x)^{m}}(C(x)-1)$. Otherwise, $\pi^{(j)}>i_{j-1}$ for all $j=2,3, \ldots, m$, which gives a contribution of $x^{m} C^{m}(x)$. Hence,

$$
G_{m}(x)=\frac{x^{m}}{(1-x)^{m}}(C(x)-1)+x^{m} C^{m}(x),
$$

for all $m \geq 3$.

We now focus on the case $m=2$. First, let $H$ be the generating function for permutations $i \pi^{\prime} n \pi^{\prime \prime} \in S_{n}(T)$ with exactly two left-right maxima and containing the subsequence $n(n-1) \cdots(i+1)$. Let us write an equation for $H$. If $i=1$, then we have a contribution of $x^{2} /(1-x)$. Otherwise, $n-1 \geq i \geq 2$ and consider the position of $i-1$. If $(i-1)$ is the first letter in $\pi^{\prime}$, then we have a contribution of $x H$. If $(i-1) \in \pi^{\prime}$ but is not the first letter in $\pi^{\prime}$, then $\pi$ must have the form $i \alpha(i-1) \beta n(n-1) \cdots(i+1)$ with $\alpha<\beta<i-1, \alpha \neq \emptyset$, and $\alpha, \beta$ both 231-avoiders, which implies a contribution of $x^{3}(C(x)-1) C(x) /(1-x)$. Otherwise, $i-1 \in \pi^{\prime \prime}$ and then $\pi$ can be decomposed as in Figure 3, where $\downarrow$ indicates a region of decreasing entries. Since $\operatorname{St}(i A n B)$ (B is spread over two

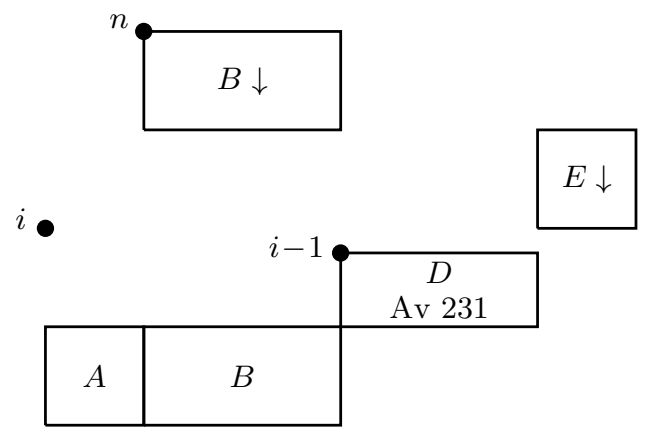

Figure 3: A permutation counted by $H$ with $i-1$ after $n$

regions) is of the type counted by $H$ and $D$ contributes $C(x)$, we get a contribution of $x /(1-x) C(x) H$. Hence,

$$
H=\frac{x^{2}}{1-x}+x H+\frac{x^{3}}{1-x}(C(x)-1) C(x)+\frac{x}{1-x} C(x) H,
$$


which leads to

$$
H=\frac{x^{2} /(1-x)+x^{3}(C(x)-1) C(x) /(1-x)}{1-x-x C(x) /(1-x)} .
$$

Now let us write an equation for $G_{2}(x)$. Let $\pi=i \pi^{\prime} n \pi^{\prime \prime}$ with exactly two left-right maxima. If $i=1$, then we have a contribution of $x^{2} C(x)$. Otherwise, $n-1 \geq i \geq 2$ and again consider the position of $i-1$. If $\pi^{\prime}=(i-1) \pi^{\prime \prime \prime}$, then we have a contribution of $x G_{2}(x)$. If $\pi^{\prime}=\alpha(i-1) \beta$ such that $\alpha$ is not empty, then $\pi=i \alpha(i-1) \beta n \pi^{\prime \prime}$ with $\alpha<\beta<i-1<\pi^{\prime \prime}<n$, which gives a contribution of $x^{3}(C(x)-1) C(x)^{2}$. Thus, we can assume that $i-1$ belongs to $\pi^{\prime \prime}$. In this case, $\pi$ can be written as $\pi=i \pi^{\prime} n \alpha^{\prime}(i-1) \alpha^{\prime \prime} \alpha^{\prime \prime \prime}$ such that $i \pi^{\prime} n \alpha^{\prime}$ has two left-right maxima and contains the subsequence $n(n-1) \cdots(j+1)$, each letter in $\alpha^{\prime \prime}$ is greater than each letter smaller than $i$ in $\pi^{\prime} \alpha^{\prime}$, and $i<\alpha^{\prime \prime \prime}<j+1$, where $\alpha^{\prime \prime}, \alpha^{\prime \prime \prime}$ avoids 231 and $i \pi^{\prime} n \alpha^{\prime}$ avoids $T$. Thus, we have a contribution of $x H C(x)^{2}$. Hence,

$$
G_{2}(x)=x^{2} C(x)+x G_{2}(x)+x^{3}(C(x)-1) C(x)^{2}+x H C(x)^{2},
$$

which implies

$$
G_{2}(x)=\frac{x^{2} C(x)+x^{3}(C(x)-1) C(x)^{2}+x H C(x)^{2}}{1-x} .
$$

Summing over $m \geq 0$, we obtain

$$
F_{T}(x)=1+x C(x)+\frac{x^{2} C(x)+x^{3}(C(x)-1) C^{2}(x)+x H C(x)^{2}}{1-x}+\frac{x^{3}(C(x)-1)}{(1-x)^{2}(1-2 x)}+\frac{x^{3} C^{3}(x)}{1-x C(x)},
$$

and this expression simplifies to the stated form.

\subsection{Case 193: $\{1324,2431,3142\}$}

Observe that each pattern here contains 132 . So if a permutation avoids 132 , then it certainly avoids $T$.

Theorem 3.19 Let $T=\{1324,2431,3142\}$. Then

$$
F_{T}(x)=\frac{x-1+\left(x^{2}-5 x+2\right) C(x)}{1-3 x+x^{2}} .
$$

Proof. Let $G_{m}(x)$ be the generating function for $T$-avoiders with $m$ left-right maxima. Clearly, $G_{0}(x)=1$ and $G_{1}(x)=x F_{T}(x)$.

Now let us write an equation for $G_{m}(x)$ where $m \geq 2$. Let $\pi=i_{1} \pi^{(1)} i_{2} \pi^{(2)} \cdots i_{m} \pi^{(m)} \in S_{n}(T)$ with exactly $m$ left-right maxima. We see that $i_{1}>\pi^{(1)}>\pi^{(2)}>\cdots>\pi^{(m-1)}$ (to avoid 1324) and $\pi^{(m)}$ can be written as $\alpha^{(1)} \alpha^{(2)} \cdots \alpha^{(m)}$ such that $\pi^{(m-1)}>\alpha^{(1)}$ and $i_{1}<\alpha^{(2)}$ (to avoid 3142) and $\alpha^{(2)}<i_{2}<\alpha^{(3)}<\cdots<i_{m-1}<\alpha^{(m)}<i_{m}$ (to avoid 2431). Furthermore, $\pi^{(j)}$ avoids 132 for all $j=1,2, \ldots, m-1$ (or $i_{m}$ is the 4 of a 1324), and at most one of $\alpha^{(2)}, \ldots, \alpha^{(m)}$ is nonempty (to avoid 1324). We consider two cases according as $\alpha^{(2)}=\cdots=\alpha^{(m)}=\emptyset$ or not:

- $\alpha^{(2)}=\cdots=\alpha^{(m)}=\emptyset$. Here, $\alpha^{(1)}$ only needs to avoid $T$ and we have a contribution of $x^{m} C(x)^{m-1} F_{T}(x)$ 
- There is a unique $j$ in the interval $[2, m]$ such that $\alpha^{(j)} \neq \emptyset$. Here, $\alpha^{(1)}$ must avoid 132 (or $\alpha^{(j)}$ contains the 4 of 1324), and $\alpha^{(j)}$ must avoid both $213 \approx 324$ (or $i_{1}$ is the 1 of a 1324) and 2431. Also, $\pi^{(j)}=\cdots=\pi^{(m)}=\emptyset$ (to avoid 3142). So $\pi^{(1)}, \ldots, \pi^{(j-1)}, \alpha^{(1)}$ each contribute $C(x)$ and we get a contribution of $x^{m} C(x)^{j}(K(x)-1)$, where $K(x)=\frac{1-2 x}{1-3 x+x^{2}}$ is the generating function for $\{213,2431\}$ avoiders [26, Seq. A001519].

Thus,

$$
G_{m}(x)=x^{m} C(x)^{m-1} F_{T}(x)+\sum_{j=2}^{m} x^{m} C(x)^{j}(K(x)-1) .
$$

Summing over $m \geq 2$ and using the expressions for $G_{1}(x)$ and $G_{0}(x)$, we obtain

$$
F_{T}(x)=1+x F_{T}(x)+\sum_{m \geq 2}\left(x^{m} C(x)^{m-1} F_{T}(x)+\sum_{j=2}^{m} x^{m} C(x)^{j}(K(x)-1)\right) .
$$

Solving for $F_{T}(x)$ gives the stated generating function after simplification.

3.10 Case 210: $\{1243,1324,2431\}$

Theorem 3.20 Let $T=\{1243,1324,2431\}$. Then

$$
F_{T}(x)=\frac{\left(1-6 x+13 x^{2}-11 x^{3}+4 x^{4}\right)}{x^{2}(1-x)^{2}} C(x)-\frac{1-6 x+12 x^{2}-8 x^{3}+2 x^{4}}{x^{2}(1-x)(1-2 x)} .
$$

Proof. Let $G_{m}(x)$ be the generating function for $T$-avoiders with $m$ left-right maxima. Clearly, $G_{0}(x)=1$ and $G_{1}(x)=x F_{T}(x)$.

For $G_{m}(x)$ with $m \geq 2$, suppose $\pi=i_{1} \pi^{(1)} i_{2} \pi^{(2)} \cdots i_{m} \pi^{(m)} \in S_{n}(T)$ with exactly $m$ left-right maxima. Since $\pi$ avoids $1324, \pi^{(s)}<i_{1}$ for all $s=1,2, \ldots, m-1$. Since $\pi$ avoids $1243, \pi^{(m)}<i_{2}$.

First, suppose $\pi^{(m)}$ has a letter greater than $i_{1}$ and consider two cases:

- all the letters between $i_{1}$ and $i_{2}$ in $\pi^{(m)}$ are increasing. Here, $\pi^{(1)} \cdots \pi^{(m-1)}$ is decreasing $(\pi$ avoids 1243$)$, and $\pi^{(m)}$ can be decomposed as ( $\pi$ avoids 2431$) \alpha\left(i_{1}+1\right) \cdots\left(i_{2}-1\right)$ and, writing $\pi^{(1)} \cdots \pi^{(m-1)}$ as $j_{s} j_{s-1} \cdots j_{1}, \alpha$ can be further decomposed as $\alpha^{(0)} \alpha^{(1)} \cdots \alpha^{(s)}$ with $\alpha^{(0)}<j_{1}<$ $\alpha^{(1)}<\cdots<j_{s}<\alpha^{(s)}<i_{1}$, where $\alpha^{(0)}$ avoids 132 and $\alpha^{(i)}$ is an increasing subword for all $i=1,2, \ldots, s$. In short, $\pi$ has the schematic form shown in Figure 4 , where $\downarrow$ denotes decreasing, $\uparrow$ denotes increasing, and blank regions are empty. If $A$ is empty, then so is $C$ and, in any case, the position of $i_{m}$ is determined by $A C$. From Figure $4, \pi$ can be written as $i_{1} A_{1} i_{m} B C D$, where $A_{1}$ consists of $A$ and the left-right maxima $i_{2}, \ldots, i_{m-1}$, and $\pi$ is determined by $\operatorname{St}\left(A_{1} C\right), \operatorname{St}(B)$, and $\operatorname{St}(D)$. The latter two contribute $C(x)$ and $\frac{x}{1-x}$, respectively. Now $A C$ is a $\{132,231\}$-avoider and $\operatorname{St}\left(A_{1} C\right)$ can be viewed as a $\{132,231\}$-avoider decorated with $m-2$ dots (for $i_{2}, \ldots, i_{m-1}$ ) placed in the spaces between its entries but not after the first ascent. For example, the 4 boxes shown accept dots in $\square 5 \square 2 \square 1 \square 34$. The generating function for such constructs with $M$ dots, each dot counting as a letter, can be shown to be $x^{M} L(x)^{M+1}$. Thus, with $M=m-2$, the contribution of this case is $\frac{x^{m+1}}{1-x} L(x)^{m-1} C(x)$. 


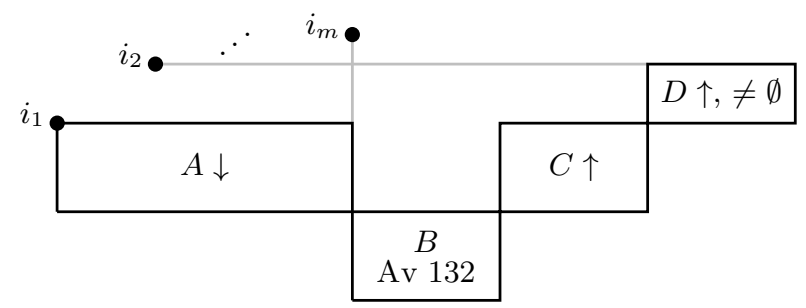

Figure 4: A $T$-avoider with at least one entry between $i_{1}$ and $i_{2}$, and these entries increasing

- the letters between $i_{1}$ and $i_{2}$ in $\pi^{(m)}$ do not form an increasing sequence, where the sequence $\pi^{(1)} \cdots \pi^{(m-1)} \alpha$ is decreasing, and $\pi^{(m)}=\alpha \beta$ with $\alpha<i_{1}<\beta<i_{2}$. Thus, we have a contribution of $\frac{x^{m}}{(1-x)^{m}}\left(L(x)-\frac{1}{1-x}\right)$.

Hence, for all $m \geq 2$,

$$
G_{m}(x)=H_{m}(x)+\frac{x^{m+1}}{1-x} L(x)^{m-1} C(x)+\frac{x^{m}}{(1-x)^{m}}\left(L(x)-\frac{1}{1-x}\right),
$$

where $H_{m}(x)$ is the generating function for those permutations $\pi \in S_{n}(T)$ with $\pi^{(m)}<i_{1}$ (in other words $\left.i_{1}=n+1-m\right)$.

Now let us write an equation for $H_{m}(x)$. Let $\pi=i_{1} \pi^{(1)} i_{2} \pi^{(2)} \cdots i_{m} \pi^{(m)} \in S_{n}(T)$ with exactly $m$ left-right maxima such that $i_{j}=n+j-m$ for all $j$. If $\pi^{(1)}=\emptyset$ then we have a contribution of $x H_{m-1}(x)$. Otherwise, if we assume that $\pi^{(1)}$ has exactly $s$ left-right maxima, it is not hard to see that those permutations are bijection with the permutations with exactly $m+s-1$ left-right maxima. Thus,

$$
H_{m}(x)=x H_{m-1}(x)+x\left(G_{m}(x)+G_{m+1}(x)+G_{m+2}(x)+\cdots\right),
$$

with $H_{1}(x)=G_{1}(x)$ by the definitions.

Define $G(x ; t)=\sum_{m \geq 0} G_{m}(x) t^{m}$ and $H(x ; t)=\sum_{m \geq 1} H_{m}(x) t^{m}$. Note that $G(x ; 1)=F_{T}(x)$. Hence, the above recurrences can be written as

$$
\begin{aligned}
& H(x ; t)=x t F_{T}(x)+x t H(x ; t)+\frac{x t^{2}}{1-t}\left(F_{T}(x)-1\right)-\frac{x t}{1-t}(G(x ; t)-1), \\
& G(x ; t)=1+H(x ; t)+\frac{x^{3} t^{2} L(x) C(x)}{(1-x)(1-x t)}+\frac{x^{2} t^{2}}{(1-x)(1-x-x t)}(L(x)-1 /(1-x)) .
\end{aligned}
$$

Thus,

$$
\begin{aligned}
\left(1+\frac{x t}{(1-t)(1-x t)}\right) G(x ; t) & =\frac{1}{1-x t}+\frac{x t}{(1-t)(1-x t)} F_{T}(x) \\
& +\frac{x^{3} t^{2} L(x) C(x)}{(1-x)(1-x t)}+\frac{x^{2} t^{2}}{(1-x)(1-x-x t)}(L(x)-1 /(1-x)) .
\end{aligned}
$$

This equation can be solved by the kernel method upon taking $t=C(x)$ which leads, after simplification, to our theorem. 


\subsection{Case 211: $\{1234,1324,2341\}$}

All three patterns contain 123 and so $T$-avoiders consist of 123 -avoiders together with $T$-avoiders that contain a 123. The former are counted by $C(x)$. To count the latter, let $a b c$ at positions $i, j, k$ be the leftmost 123 pattern (smallest $i$, then smallest $j$, then smallest $k$ ). This 123 pattern divides the permutation diagram into rectangular regions as shown in Figure 5 where the bullets represent the leftmost 123. Shaded regions are empty for the indicated reason, where (min) refers to the minimal,

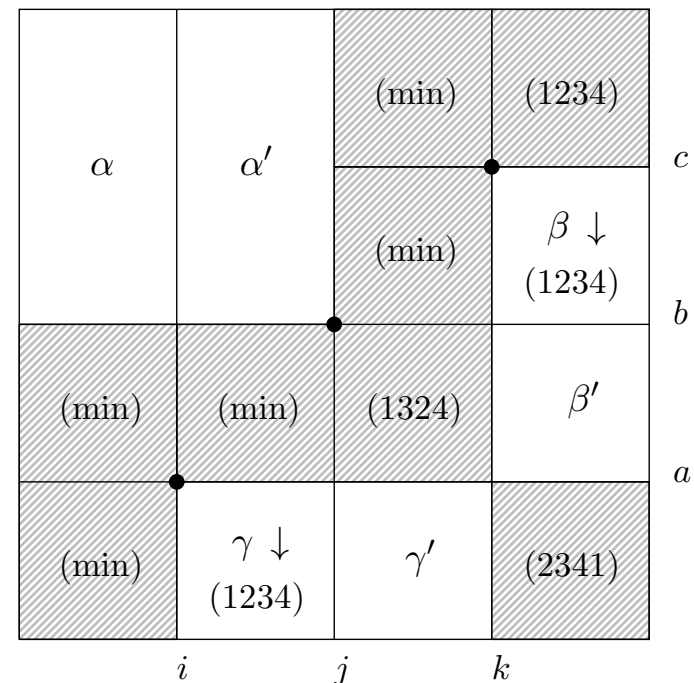

Figure 5: The leftmost (min) 123 in a $T$-avoider

that is, leftmost property of the 123 pattern, and unshaded regions are labeled $\alpha, \alpha^{\prime}$, etc. A down arrow $(\downarrow)$ indicates necessarily decreasing entries, again for the indicated reason. Also, $\alpha \alpha^{\prime}$ avoids 123 (or the middle bullet ends a 2341), while $\beta$ lies to the left of $\beta^{\prime}$ and $\gamma>\gamma^{\prime}$ (both to avoid 1324). We consider four cases based on whether $\beta^{\prime}$ or $\gamma^{\prime}$ is empty or not:

- $\beta^{\prime}, \gamma^{\prime}$ both empty. Here, $\operatorname{St}\left(\alpha a \alpha^{\prime} c\right)$ is a 123 -avoider $\tau$ of length $\geq 2$ with last entry $>1$. Say $\tau$ has length $n$, with 1 in position $i<n$ and last entry $\ell \geq 2$. Now $\beta$ serves to "decorate" $\tau$ with 0 or more dots inserted arbitrarily in the $\ell-1$ spaces between 1 and $\ell$. Similarly, $\gamma$ amounts to 0 or more dots inserted in the $n-i$ spaces between $i$ and $n$. Thus, the contribution of this case is

$$
x \sum_{n \geq 2} \sum_{i=1}^{n-1} \sum_{\ell=2}^{n} C(n, i, \ell) x^{n} \sum_{u, v \geq 0}\left(\begin{array}{c}
u+\ell-2 \\
u
\end{array}\right)\left(\begin{array}{c}
v+n-i-1 \\
v
\end{array}\right),
$$

where $C(n, i, \ell)$ is the number of 123 -avoiders of length $n$ with 1 in position $i$ and last entry $\ell$. It is known that $C(n, i, \ell)=\frac{n-2-i+\ell}{n+i-\ell}\left(\begin{array}{c}n+i-\ell \\ n-1\end{array}\right)$ (see [14]), a generalized Catalan number, and the displayed sum evaluates to the compact expression $x^{3} C(x)^{5}$.

- $\beta^{\prime}$ empty, $\gamma^{\prime}$ nonempty. Here, $\gamma^{\prime}$ avoids 123 and 132 and so the contribution is

$$
x^{3} C(x)^{5}(L-1)=x^{3} C(x)^{5} \frac{x}{1-2 x} .
$$


- $\beta^{\prime}$ nonempty, $\gamma^{\prime}$ empty. Here, $\beta^{\prime}$ avoids 123 and 213 and so the contribution is

$$
x^{3} C(x)^{5}(L-1)=x^{3} C(x)^{5} \frac{x}{1-2 x} .
$$

- $\beta^{\prime}, \gamma^{\prime}$ both nonempty. In case $\gamma^{\prime}$ is decreasing, the only restriction on $\beta^{\prime}$ is to avoid 123 and 213 (since $\gamma^{\prime} \neq \emptyset$ ). So $\gamma^{\prime}$ contributes $\frac{x}{1-x}$ and $\beta^{\prime}$ contributes $L-1$. In case $\gamma^{\prime}$ is not decreasing, $\beta^{\prime}$ is decreasing (to avoid 1234) and $\gamma^{\prime}$ contributes $L-\frac{1}{1-x}$ while $\beta^{\prime}$ contributes $\frac{x}{1-x}$. So, $\beta^{\prime}, \gamma^{\prime}$ together contribute $\frac{x}{1-x}(L-1)+\left(L-\frac{1}{1-x}\right) \frac{x}{1-x}=\frac{x^{2}}{(1-x)^{2}(1-2 x)}$ for an overall contribution of $x^{3} C(x)^{5} \frac{x^{2}}{(1-x)^{2}(1-2 x)}$.

Hence, summing over 123-avoiders and the four cases,

$$
F_{T}(x)=C(x)+x^{3} C(x)^{5}\left(1+\frac{x}{1-2 x}+\frac{x}{1-2 x}+\frac{x^{2}}{(1-x)^{2}(1-2 x)}\right) .
$$

Simplifying this expression, we have established the following result.

Theorem 3.21 Let $T=\{1234,1324,2341\}$. Then

$$
F_{T}(x)=\frac{\left(1-4 x+5 x^{2}-3 x^{3}\right) C(x)-\left(1-4 x+6 x^{2}-4 x^{3}\right)}{x(1-x)^{2}(1-2 x)} .
$$

\subsection{Case 212: $\{1324,2413,2431\}$}

Note that each pattern contains 132 .

Theorem 3.22 Let $T=\{1324,2413,2431\}$. Then

$$
F_{T}(x)=1+\frac{x\left(1-4 x+4 x^{2}-x^{3}-x\left(1-4 x+2 x^{2}\right) C(x)\right)}{\left(1-3 x+x^{2}\right)\left(1-3 x+x^{2}-x(1-2 x) C(x)\right)} .
$$

Proof. Let $G_{m}(x)$ be the generating function for $T$-avoiders with $m$ left-right maxima. Clearly, $G_{0}(x)=$ 1 and $G_{1}(x)=x F_{T}(x)$. For $m=2, \pi$ has the form $i \alpha n \beta$ with $\alpha<i$, and $\beta$ cannot contain both letters $>i$ and $<i$ (2413 and 2431). If $\beta$ has no letter $>i$, then $i=n-1$ and deleting it gives a contribution of $x\left(F_{T}(x)-1\right.$ ). Otherwise, $\beta>i$ and $\alpha$ avoids 132 (due to $n$ ) and $\beta$ avoids both 213 (due to $i$ ) and 2431 and is nonempty, contributing $x^{2} C(x)(K(x)-1)$, where $K(x)=\frac{1-2 x}{1-3 x+x^{2}}$ is the generating function for $\{213,2431\}$-avoiders [26, Seq. A001519]. So $G_{2}(x)=x\left(F_{T}(x)-1\right)+x^{2} C(x)(K(x)-1)$.

Now let us write equation for $G_{m}(x)$ for $m \geq 3$. Suppose $\pi=i_{1} \pi^{(1)} i_{2} \pi^{(2)} \cdots i_{m} \pi^{(m)} \in S_{n}(T)$ with exactly $m$ left-right maxima. Since $\pi$ avoids 1324 , we have $\pi^{(s)}<i_{1}$ for all $s=1,2, \ldots, m-1$. Since $\pi$ avoids 2413 and 2431, either $\pi^{(2)}$ is empty or $\pi^{(m)}$ has no letter between $i_{1}$ and $i_{2}$ (or both). Thus, we have three cases

- $\pi^{(2)}=\emptyset$ and $\pi^{(m)}$ has no letter between $i_{1}$ and $i_{2}$. Here, $i_{2}$ and its position is determined by the rest of the avoider, and we have a contribution of $x G_{m-1}(x)$. 
- $\pi^{(2)}=\emptyset$ and $\pi^{(m)}$ has a letter between $i_{1}$ and $i_{2}$. Here, $\pi^{(3)}=\cdots=\pi^{(m-1)}=\emptyset$ (or they would contain the 1 of a 2413), and since $\pi$ avoids both 2413 and 2431, $i_{1}<\pi^{(m)}$. Additionally, $\pi^{(m)}$ is not empty and avoids both 213 (or $i_{1}$ is the 1 of a 1324) and 2431, and $\pi^{(1)}$ avoids 132 (or $i_{m}$ is the 4 of a 1324). Hence, the contribution is $x^{m} C(x)(K(x)-1)$.

- $\pi^{(2)} \neq \emptyset$ and $\pi^{(m)}$ has no letter between $i_{1}$ and $i_{2}$. Since $\pi$ avoids 2413 , we see that $i_{1}>$ $\pi^{(1)}>\pi^{(s)}$ for all $s=2,3, \ldots, m-1$. Also, $\pi^{(1)}$ avoids 132 and $i_{2}=i_{1}+1$. So $\pi$ can be recovered from $\operatorname{St}\left(i_{1} \pi^{(1)}\right)$ and $\operatorname{St}\left(i_{2} \pi^{(2)} \cdots i_{m} \pi^{(m)}\right)$, giving respective contributions of $x C(x)$ and $G_{m-1}(x)-G_{m-1}^{\prime}(x)$ where $G_{m-1}^{\prime}(x)$ counts the $\pi^{(2)}$ empty case, that is, $G_{m}^{\prime}(x)$ is the generating function for $T$-avoiders with $m$ left-right maxima in which the first letter is smaller than the second. Thus, the contribution is $x C(x)\left(G_{m-1}(x)-G_{m-1}^{\prime}(x)\right)$.

Hence, for all $m \geq 3$,

$$
G_{m}(x)=x G_{m-1}(x)+x^{m} C(x)(K(x)-1)+x C(x)\left(G_{m-1}(x)-G_{m-1}^{\prime}(x)\right) .
$$

For $G_{2}^{\prime}(x)$, a $T$-avoider has the form $\pi=i n \pi^{\prime}$. If $\pi^{\prime}$ is empty, the contribution is $x^{2}$. So suppose $\pi^{\prime} \neq \emptyset$. If $i=n-1$, then we have $x^{2}\left(F_{T}(x)-1\right)$, but if $i<n-1$, then since $\pi$ avoids 2413 and 2431, we see that $i=1$ and $\pi=1 n \pi^{\prime}$ with $\pi^{\prime} \neq \emptyset$ avoiding 213 and 2431, giving a contribution of $x^{2}(K(x)-1)$. So

$$
G_{2}^{\prime}(x)=x^{2}\left(K(x)+F_{T}(x)-1\right) .
$$

For $G_{m}^{\prime}(x)$ with $m \geq 3$, using the same three cases as above, we find by similar arguments the recurrence

$$
\begin{aligned}
G_{m}^{\prime}(x) & =x G_{m-1}^{\prime}(x)+x^{m}(K(x)-1)+x\left(G_{m-1}(x)-G_{m-1}^{\prime}(x)\right) \\
& =x^{m}(K(x)-1)+x G_{m-1}(x) .
\end{aligned}
$$

Substituting (6) and (77) into (5), we have

$$
G_{m}(x)=x(C(x)+1) G_{m-1}(x)-x^{2} C(x) G_{m-2}(x)
$$

for $m \geq 3$, with $G_{2}, G_{1}, G_{0}$ as above. Summing over $m \geq 3$, we obtain

$$
F_{T}(x)-1-x F_{T}(x)-G_{2}(x)=x(C(x)+1)\left(F_{T}(x)-1-x F_{T}(x)\right)-x^{2} C(x)\left(F_{T}(x)-1\right) .
$$

Solving for $F_{T}(x)$ completes the proof.

For the next two cases, we set $a_{T}(n)=\left|S_{n}(T)\right|$ and let $a_{T}\left(n ; j_{1}, j_{2}, \ldots, j_{s}\right)$ denote the number of permutations in $S_{n}(T)$ whose first $s$ letters are $j_{1} j_{2} \cdots j_{s}$.

\subsection{Case 231: $\{1324,1342,2341\}$}

Set $b(n ; j)=a_{T}(n ; j, j+1)$.

LEMMA 3.23 For $1 \leq j \leq n-2$,

$$
a_{T}(n ; j)=a_{T}(n-1 ; 1)+\cdots+a_{T}(n-1 ; j)+b(n ; j)
$$

and $a_{T}(n ; n)=a_{T}(n ; n-1)=a_{T}(n ; n-2)=a_{T}(n-1)$. 
Proof. The initial conditions $a_{T}(n ; n)=a_{T}(n ; n-1)=a_{T}(n-1)$ easily follow from the definitions. For $1 \leq j \leq n-2$, we have

$$
a_{T}(n ; j)=\sum_{i=1}^{j-1} a_{T}(n ; j, i)+\sum_{i=j+1}^{n} a_{T}(n ; j, i) .
$$

So assume $1 \leq j \leq n-2$ and let $\pi=j i \pi^{\prime}$ be a member of $S_{n}(T)$. We consider several cases on $i$. Since $\pi$ avoids 1324 and 1342, we have that either $1 \leq i \leq j+1$ or $i=n$. If $i=n$, then $\pi$ avoids $T$ if and only if $j \pi^{\prime}$ avoids $T$, so $a_{T}(n ; j, n)=a_{T}(n-1, j)$. So,

$$
a_{T}(n ; j)=\sum_{i=1}^{j-1} a_{T}(n ; j, i)+a_{T}(n-1 ; j)+b(n ; j) .
$$

Let $1 \leq i \leq j-1$. If $\pi$ avoids $T$, then $i \pi^{\prime}$ avoids $T$. On other hand, if $\pi$ contains 1324 (resp. 1342), then $i \pi^{\prime}$ contains 1324 (resp. 1324). Also, if $\pi$ contains 2341 where $j$ does not occur in the corresponding occurrence, then $i \pi^{\prime}$ contains 2341. Thus, we assume that $\pi$ contains $j a b c$ with $c<j<a<b$. If $c<i$, then $i \pi^{\prime}$ contains 2341, otherwise $i \pi^{\prime}$ contains 1342. Therefore, $i \pi^{\prime}$ avoids $T$ if and only if $\pi$ avoids $T$, which implies $a_{T}(n-1 ; j, i)=a_{T}(n ; i)$. Hence,

$$
a_{T}(n ; j)=\sum_{i=1}^{j-1} a_{T}(n-1 ; i)+a_{T}(n-1 ; j)+b(n ; j),
$$

as required.

Define $A_{T}(n ; v)=\sum_{j=1}^{n} a_{T}(n ; j) v^{j-1}$ and $B(n ; v)=\sum_{j=1}^{n-2} b(n ; j) v^{j-1}$. Then Lemma 3.23 can be written as

$$
A_{T}(n ; v)=\frac{1}{1-v}\left(A_{T}(n-1 ; v)-v^{n} A_{T}(n-1 ; 1)\right)+B(n ; v)
$$

with $A_{T}(0 ; v)=A_{T}(1 ; v)=1$.

Define $A_{T}(x, v)=\sum_{n \geq 0} A_{T}(n ; v) x^{n}$ and $B(x, v)=\sum_{n \geq 3} B(n ; v) x^{n}$. Then, the above recurrence can be written as

$$
A(x, v)=1+\frac{x}{1-v}(A(x, v)-v A(x v, 1))+B(x, v) .
$$

LEMma 3.24 We have $B(x, v)=\frac{x^{3}(1-2 x v)}{\left(1-3 x v+x^{2} v^{2}\right)(1-2 x)}$.

Proof. Let $\pi=j(j+1) \pi^{\prime} \in S_{n}(T)$ with $1 \leq j \leq n-2$. Since $\pi$ avoids 2341, we can express $\pi$ as $\pi=j(j+1) \alpha \beta$ with $\alpha<j<j+1<\beta$. Note that $\pi$ avoids $T$ if and only if $\alpha$ avoids both 132,2341 and $\beta$ avoids both 213,231. By [26, Seq. A001519] we have that $F_{\{132,2341\}}(x)=\frac{1-2 x}{1-3 x+x^{2}}$, and by [25] we have that $F_{\{213,231\}}(x)=L(x)$, yielding

$$
\begin{aligned}
B(x, v) & =\sum_{n \geq 3} \sum_{j=1}^{n-2}\left|S_{j-1}(132,2341) \| S_{n-1-j}(213,231)\right| v^{j-1} x^{n} \\
& =x^{2} F_{\{132,2341\}}(x v)\left(F_{\{213,231\}}(x)-1\right),
\end{aligned}
$$


which leads to $B(x, v)=\frac{x^{3}(1-2 x v)}{\left(1-3 x v+x^{2} v^{2}\right)(1-2 x)}$, as required.

By (8) and Lemma 3.24, we obtain

$$
A(x / v, v)=1+\frac{x}{v(1-v)}(A(x / v, v)-v A(x, 1))+\frac{x^{3}(1-2 x)}{v^{2}\left(1-3 x+x^{2}\right)(v-2 x)} .
$$

To solve the preceding functional equation, we apply the kernel method and take $v=\frac{1+\sqrt{1-4 x}}{2}=$ $1 / C(x)$. Then

$$
F_{T}(x)=A(x, 1)=C(x)+\frac{x^{3}(1-2 x) C^{4}(x)}{\left(1-3 x+x^{2}\right)(1-2 x C(x))} .
$$

After simplification, this gives the following result.

Theorem 3.25 Let $T=\{1324,1342,2341\}$. Then

$$
F_{T}(x)=\frac{(1-3 x)(1-2 x-x C(x))}{(1-4 x)\left(1-3 x+x^{2}\right)} .
$$

\subsection{Case 241: $\{1324,1243,1234\}$}

Set $T=\{1324,1243,1234\}$ and $b(n ; j)=a_{T}(n ; j, n-1)$.

Lemma 3.26 For $1 \leq j \leq n-2$,

$$
\begin{aligned}
a_{T}(n ; j) & =a_{T}(n-1 ; 1)+\cdots+a_{T}(n-1 ; j)+b(n ; j), \\
b(n ; j) & =b(n-1 ; 1)+\cdots+b(n-1 ; j-1)+a_{T}(n-2 ; j)
\end{aligned}
$$

and $a_{T}(n ; n)=a_{T}(n ; n-1)=a_{T}(n ; n-2)=a_{T}(n-1), b(n ; n)=b(n ; n-2)=a_{T}(n-2)$ and $b(n ; n-1)=0$.

Proof. The initial conditions $a_{T}(n ; n)=a_{T}(n ; n-1)=a_{T}(n ; n-2)=a_{T}(n-1), b(n ; n)=b(n ; n-2)=$ $a_{T}(n-2)$ and $b(n ; n-1)=0$ easily follow from the definitions. For $1 \leq j \leq n-2$, we have

$$
a_{T}(n ; j)=\sum_{i=1}^{j-1} a_{T}(n ; j, i)+\sum_{i=j+1}^{n} a_{T}(n ; j, i) .
$$

So assume $1 \leq j \leq n-3$ and let $\pi=j i \pi^{\prime}$ be a member of $S_{n}(T)$. We consider several cases on $i$. If $i=n$, then $a_{T}(n ; j, n)=a_{T}(n-1 ; j)$. If $1 \leq i<j$, then $j i \pi^{\prime}$ (respectively, $j n \pi^{\prime}$ ) avoids $T$ if and only if $i \pi^{\prime}$ (respectively, $j \pi^{\prime}$ ) avoids $T$, so $a_{T}(n ; j, i)=a_{T}(n-1 ; i)$ for all $i=1,2, \ldots, j-1$ and $a_{T}(n ; j, n)=a_{T}(n-1 ; j)$. Since $\pi$ avoids 1234 and 1243, we see that either $i<j$ or $j>n-2$, and $a_{T}(n ; j, n)=a_{T}(n-1 ; j)$. Thus,

$$
a_{T}(n ; j)=\sum_{i=1}^{j-1} a_{T}(n-1 ; i)+a_{T}(n-1 ; j)+b(n ; j),
$$

which completes the proof of the first recurrence relation. 
For the second relation, by similar reasons, we have

$$
b(n ; j)=\sum_{i=1}^{j-1} a_{T}(n ; j, n-1, i)+\sum_{i=j+1}^{n-2} a_{T}(n ; j, n-1, i)+a_{T}(n ; j, n-1, n) .
$$

Clearly, $a_{T}(n ; j, n-1, n)=a_{T}(n-2, j)$. The permutation $j(n-1)(n-2) \pi^{\prime \prime}$ contains 1324 , so $a_{T}(n ; j, n-1, n-2)=0$. The permutation $j(n-1) i \pi^{\prime \prime}$ with $j+1 \leq i \leq n-3$ contains either 1234 or 1243 , so $a_{T}(n ; j, n-1, i)=0$. Thus,

$$
b(n ; j)=\sum_{i=1}^{j-1} a_{T}(n ; j, n-1, i)+a_{T}(n-2 ; j) .
$$

Let $j(n-1) i \pi^{\prime \prime} \in S_{n}$. If $i(n-1) \pi^{\prime \prime}$ contains a pattern in $T$, then $j(n-1) i \pi^{\prime \prime}$ contains the same pattern in $T$. Now, suppose $i(n-1) \pi^{\prime \prime}$ avoids $T$, so if $\pi=j(n-1) i \pi^{\prime \prime}$ contains 1234 or 1243 , then $i(n-1) \pi^{\prime \prime}$ contains 1234 or 1243 , which implies that $\pi$ avoids 1234 and 1243. If $\pi$ contains 1324, then we can assume that 1324 occurs in $\pi$ as a subsequence $j a b c$ with $j<b<a<c$, otherwise $i(n-1) \pi^{\prime}$ contains 1324. Also, we can assume that $b=n-1$ which gives $c=n$, otherwise $i a b c$ occurs in $i(n-1) \pi^{\prime \prime}$. Since $j \leq n-3, \pi$ has an element $d$ such that either $j<d<b$ or $b<d<n-1$. Thus, $i(n-1) \pi^{\prime \prime}$ contains either $i d b a$ or $i b d a$ or $i b a d$, that is $i(n-1) \pi^{\prime \prime}$ does not avoid $T$. Therefore, $\pi$ avoids $T$. Hence,

$$
a_{T}(n ; j, n-1, i)=a_{T}(n-1 ; i, n-2)=b(n-1 ; i),
$$

for all $i=1,2, \ldots, j-1$, which implies

$$
b(n ; j)=\sum_{i=1}^{j-1} b(n-1 ; i)+a_{T}(n-2 ; j) .
$$

This completes the proof.

Define $A_{T}(n ; v)=\sum_{j=1}^{n} a_{T}(n ; j) v^{j-1}$ and $B(n ; v)=\sum_{j=1}^{n} b(n ; j) v^{j-1}$. Then Lemma 3.26 can be written as

$$
\begin{aligned}
A_{T}(n ; v) & =A_{T}(n-1 ; 1)\left(v^{n-1}+v^{n-2}+v^{n-3}\right)+\frac{1}{1-v}\left(A_{T}(n-1 ; v)-v^{n-3} A_{T}(n-1 ; 1)\right) \\
& +B(n ; v)-A_{T}(n-2 ; 1) v^{n-1}, \\
B(n ; v) & =A_{T}(n-2 ; 1)\left(v^{n-1}+v^{n-3}\right)+\frac{1}{1-v}\left(v B(n-1 ; v)-B(n-1 ; 1) v^{n-3}\right) \\
& +A_{T}(n-2 ; v)+A_{T}(n-3 ; 1) v^{n-2},
\end{aligned}
$$

with $A_{T}(0 ; v)=A_{T}(1 ; v)=1, A_{T}(2 ; v)=1+v, B(0 ; v)=B(1 ; v)=0$ and $B(2 ; v)=v$.

Define $A_{T}(x, v)=\sum_{n \geq 0} A_{T}(n ; v) x^{n}$ and $B(x, v)=\sum_{n \geq 0} B(n ; v) x^{n}$. Then, the above recurrence 
can be written as

$$
\begin{aligned}
A_{T}(x, v) & =1+\left(x+\frac{x}{v}+\frac{x}{v^{2}}\right) A_{T}(x v, 1) \\
& +\frac{x}{1-v}\left(A_{T}(x, v)-\frac{1}{v^{2}} A_{T}(x v, 1)\right)+B(x, v)-x^{2} v A_{T}(x v, 1), \\
B(x, v) & =\frac{x}{1-v}\left(v B(x, v)-\frac{1}{v^{2}} B(x v, 1)\right)+x^{3} v A_{T}(x v, 1) \\
& +\frac{x^{2}(1+v)}{v}\left(A_{T}(x v, 1)-1\right)+x^{2} v A_{T}(x v, 1) .
\end{aligned}
$$

By multiplying (10) by $v^{2}(1-v)^{2}\left(1-\frac{x v}{1-v}\right)$ and (9) by $v^{2}(1-v)^{2}$, then adding the results, we obtain

$$
\begin{aligned}
K(x, v) A_{T}(x / v ; v) & =\frac{x\left(v^{3}(1-x-v)-x(1-v)(1-v+x v)\right)}{v} A_{T}(x ; 1)+\frac{x(1-v)}{v} B(x ; 1) \\
& +\frac{(1-v)\left(x^{2}\left(1-v^{2}\right)-v^{3}(1-x-v)\right)}{v}
\end{aligned}
$$

where $K(x, v)=x v\left(1-v^{2}\right)+x^{2}\left(1-3 v+v^{2}\right)-v^{2}(1-v)^{2}$.

To solve the preceding functional equation, we apply the kernel method and take

$$
\begin{aligned}
& v=v_{-}=\frac{2+(\sqrt{5}-1) x+\sqrt{4-12 x+6 x^{2}-4 \sqrt{5} x-2 \sqrt{5} x^{2}}}{4} \text { and } \\
& v=v_{+}=\frac{2-(\sqrt{5}+1) x+\sqrt{4-12 x+6 x^{2}+4 \sqrt{5} x+2 \sqrt{5} x^{2}}}{4},
\end{aligned}
$$

which satisfy $K\left(x ; v_{+}\right)=K\left(x, v_{-}\right)=0$. Hence, since $F_{T}(x)=A_{T}(x, 1)$, we obtain the following result. Theorem 3.27 Let $T=\{1324,1243,1234\}$. Then $F_{T}(x)$ is given by

$$
\frac{\left(v_{-}-1\right)\left(v_{+}-1\right)\left(\left(v_{-}+v_{+}\right)\left(v_{-}^{2}+v_{+}^{2}-x^{2}\right)+(x-1)\left(v_{-}^{2}+v_{+}^{2}+v_{-} v_{+}\right)\right)}{x-\left(v_{-}-1\right)\left(v_{+}-1\right)\left(v_{-}^{2}+v_{+}^{2}+v_{-} v_{+}+x\left(v_{-}+v_{+}+2-x\right)\right)} .
$$

\section{References}

[1] Enumerations of specific permutation classes, Published electronically at https://en.wikipedia.org/wiki/Enumerations_of_specific_permutation_classes .

[2] M. H. Albert, C. Homberger, J. Pantone, N. Shar and V. Vatter, Generating permutations with restricted containers, preprint, at https://arxiv.org/abs/1510.00269, 2015.

[3] M. H. Albert, S. Linton And N. RušKuc, The insertion encoding of permutations, Electron. J. Combin., 12 (2005) \#R47. 
[4] C. Banderier, M. Bousquet-Mélou, A. Denise, P. Flajolet, D. Gardy and D. Gouyou-Beauchamps, Generating functions for generating trees, Discrete Math., 246 (2002) 29-55.

[5] D. Callan, T. Mansour and M. Shattuck, Wilf classification of triples of 4-letter patterns, preprint, at http://arxiv.org/abs/1605.04969, 2016.

[6] D. Callan and Mansour, Five subsets of permutations enumerated as weak sorting permutations, Southeast Asian Bull. Math., to appear.

[7] D. Callan and Mansour, A Wilf class composed of 7 symmetry classes of triples of 4-letter patterns, Journal of Analysis \& Number Theory, 5 (2017) 19-26.

[8] D. Callan and Mansour, On permutations avoiding 1243, 2134, and another 4-letter pattern, Pure Math. Appl. (PU.M.A.), 26 (2017) 11-21.

[9] D. Callan and Mansour, On permutations avoiding 1324, 2143, and another 4-letter pattern, Pure Math. Appl. (PU.M.A.), 26 (2017) 1-10.

[10] D. Callan and Mansour, Enumeration of small Wilf classes avoiding 1324 and two other 4-letter patterns, at http://arxiv.org/abs/1705.00933, 2017.

[11] D. Callan, T. Mansour And M. Shattuck, Twelve subsets of permutations enumerated as maximally clustered permutations, Ann. Math. Inform., 47 (2017) 41-74.

[12] D. Callan, T. Mansour and M. Shattuck, Wilf classification of triples of 4-letter patterns I, Discrete Math. Theoret. Comput. Sci., 19:1 (2017) \#5, 35 pp.

[13] D. Callan, T. Mansour and M. Shattuck, Wilf classification of triples of 4-letter patterns II, Discrete Math. Theoret. Comput. Sci., 19:1 (2017) \#6, 44 pp.

[14] G. Firro And T. Mansour, Three-letter-pattern avoiding permutations and functional equations, Electron J. Combin., 13 (2006) \#R51.

[15] S. Heubach and T. Mansour, Combinatorics of Compositions and Words, CRC Press, Boca Raton, FL, 2009.

[16] Q. Hou And T. Mansour, Kernel method and linear recurrence system, J. Comput. Appl. Math., 261 (2008) 227-242.

[17] D. E. Knuth, The Art of Computer Programming, 3rd edition, Addison Wesley, Reading, MA, 1997.

[18] D. Kremer And W. C. ShiU, Finite transition matrices for permutations avoiding pairs of length four patterns, Discrete Math., 268 (2003) 171-183.

[19] W. Kuszmaul, Fast algorithms for finding pattern avoiders and counting pattern occurrences in permutations, preprint, at https://arxiv.org/abs/1509.08216, 2015. 
[20] I. LE, Wilf classes of pairs of permutations of length 4, Electron. J. Combin., 12 (2005) \#R25.

[21] P. A. Macmahon, Combinatory Analysis, Volumes 1 and 2, Cambridge University Press, 1915-16.

[22] T. Mansour, Combinatorics of Set Partitions, CRC Press, Boca Raton, FL, 2012.

[23] T. Mansour and M. Shattuck, Nine classes of permutations enumerated by binomial transform of Fine's sequence, Discrete Appl. Math., 226 (2017) 94-105.

[24] T. Mansour and A. Vainshtein, Restricted 132-avoiding permutations, Adv. in Appl. Math., 26 (2001) 258-269.

[25] R. Simion And F. W. Schmidt, Restricted permutations, European J. Combin., 6 (1985) 383-406.

[26] N. J. A. Sloane, The On-Line Encyclopedia of Integer Sequences, published electronically at http://oeis.org/.

[27] Z. E. Stankova, Forbidden subsequences, Discrete Math., 132 (1994) 291-316.

[28] Z. Stankova, Classification of forbidden subsequences of length four, European J. Combin., 17 (1996) 501-517.

[29] V. VATTER, Finding regular insertion encodings for permutation classes, J. Symbolic Comput., 47 (2012) 259-265.

[30] J. West, Generating trees and the Catalan and Schröder numbers, Discrete Math., 146 (1995) 247-262. 